\title{
A NEW METHOD FOR THE IDENTIFICATION OF NON-GAUSSIAN LINE PROFILES IN ELLIPTICAL GALAXIES
}

\author{
ROELAND P. VAN DER MAREL \\ Sterrewacht Leiden, Postbus 9513, 2300 RA Leiden, The Netherlands
}

AND

MARIJN FRANX ${ }^{1}$

Harvard-Smithsonian Center for Astrophysics, 60 Garden Street, Cambridge, MA 02138

Received 1992 June 24 ; accepted 1992 October 15

\begin{abstract}
It is usually assumed that the line profiles (i.e., the distributions of stars over line-of-sight velocities) of elliptical galaxies have Gaussian shapes, characterized by a line strength $\gamma$, mean radial velocity $V$, and velocity dispersion $\sigma$. We relax this unnecessarily restrictive assumption and propose a decomposition of the line profile into orthogonal functions: the Gauss-Hermite series. This series naturally leads to two extra parameters that measure deviations of the line profile from a Gaussian: a parameter $h_{3}$ measuring asymmetric deviations and a parameter $h_{4}$ measuring symmetric deviations.

Model calculations for the outer parts of spherical galaxies yield line profiles that can deviate significantly from Gaussians. Even for models with only mild velocity dispersion anisotropy the rms deviations from a Gaussian can be of order $10 \%$. Approximating these line profiles by Gaussians yields systematic errors in the estimates of the mean radial velocity and velocity dispersion of $10 \%$ or more.

The new method is used to derive line profiles for the elliptical galaxies IC 1459, NGC 1374, and NGC 4278. All three galaxies have asymmetric line profiles on the major axis, similar to those found earlier in galaxies with kinematically distinct cores. In addition we find evidence for symmetric deviations from a Gaussian. By fitting Gaussians to these asymmetric line profiles the amplitude of the rotation curve can be overestimated by $30 \%$ or more. The results for $h_{3}$ and $h_{4}$ are not strongly dependent on the spectral resolution of the observations. The results confirm the notion that elliptical galaxies have complex structures, due to their complex formation history. It is expected that accurate measurements of line profiles will provide additional constraints on models of galaxy structure and formation.
\end{abstract}

Subject headings: galaxies: elliptical and lenticular, $\mathrm{cD}$ - galaxies: kinematics and dynamics — line: profiles

\section{INTRODUCTION}

Observed galaxy spectra are usually assumed to be the convolution of a suitably chosen template spectrum and a broadening function. This broadening function corresponds to the distribution of stars over line-of-sight velocities, henceforth referred to as the line profile. The line profile is usually assumed to be Gaussian. This gives a convenient fit to most observed spectra, in spite of the fact that the infinite wings of a Gaussian line profile imply a system of infinite mass. A variety of methods exists to determine the best fitting Gaussian broadening function, e.g., the Fourier quotient (Simkin 1974; Sargent et al 1977), the cross correlation (Tonry \& Davis 1979), the Fourier correlation quotient (Bender 1990), the Fourier fitting (Franx, Illingworth, \& Heckman 1989a, hereafter FIHa), and the direct fitting (Rix \& White 1992, hereafter RW) method. Most of our current understanding of the dynamics of elliptical galaxies (for a review see, e.g., de Zeeuw \& Franx 1991) is based on estimates of rotation velocities and velocity dispersions obtained in this way.

Unfortunately models of elliptical galaxies are not uniquely constrained by knowledge of rotation velocities and velocity dispersions alone. For example, both tangential velocity dispersion anisotropy and the presence of a dark halo can cause the observed velocity dispersion to remain more-or-less constant out to one effective radius. Also, weak or rather face-on disks in elliptical galaxies are very difficult to detect from knowledge of surface photometry, rotation velocities, and velocity dispersions alone (Rix \& White 1990). In cases like these, the different models can be distinguished by the shape of their line profiles. Tangentially anisotropic models of the outer parts of elliptical galaxies have line profiles that are more flat-topped than those of radially anisotropic models (Dejonghe 1987; Gerhard 1991; Winsall \& Freeman 1993a). The presence of a disk leads to asymmetric line profiles.

Several methods have been developed to test whether observed line profiles deviate from Gaussians (Franx \& Illingworth 1988, hereafter FI; Bender 1990; RW; Winsall \& Freeman 1993b). Significantly non-Gaussian line profiles were reported in the cores of several bright, nearby elliptical galaxies. The deviations from a Gaussian are in the sense that the line profile is asymmetric, with the asymmetry changing sign upon going from one side of the nucleus to the other. These line profiles are well fitted with the sum of two Gaussians, one component with low rotation and high dispersion, and one component with high rotation and low dispersion. These components are tentatively identified with the main body of the galaxy, and with a more rapidly rotating core component

\footnotetext{
${ }^{1}$ Visiting Astronomer at the Kitt Peak National Observatory and the Cerro Tololo Inter-American Observatory, operated by AURA, Inc., under contract to the National Science Foundation.
} 
(sometimes counter-rotating with respect to the main body of the galaxy), respectively. Such core components are most likely produced by mergers or starbursts (FI; Balcells 1991).

Now that it is possible to detect deviations of line profiles from Gaussians, one should address the question how such deviations are best quantified. A decomposition into two Gaussians is one possible approach, and seems the most logical thing to do if one has an a priori reason to believe that two kinematically distinct components are present. If not, however, the results are difficult to interpret, the more so since the errors in the six parameters of a two-Gaussian decomposition are highly correlated. From a mathematical and physical point of view one would rather like to know, e.g., the (third and) higher moments of the line profile. These can easily be compared to models, since they are calculable for any given distribution function. Furthermore they obey higher order virial equations (Merrifield \& Kent, 1990; Kent 1990), and can be used to constrain the shape of the potential (Dejonghe \& Merritt 1992). Unfortunately the higher moments of an observed line profile cannot be determined, since the wings of the line profile are ill-constrained by the observations (broad wings are hard to discriminate from differences in continuum shape between galaxy and template spectrum).

In this paper a new method is presented to parameterize the line profiles of elliptical galaxies. The observed line profile is expanded as a sum of orthogonal functions in a Gauss-Hermite series. The approach exploits the fact that Gaussians are good low-order approximations to most realistic line profiles, and naturally leads to two parameters describing deviations from a Gaussian: a parameter $h_{3}$ describing asymmetric deviations, and a parameter $h_{4}$ describing symmetric deviations. The choice of an orthogonal set of functions minimizes correlations between the errors in the parameters that describe the line profile.

In $\S 2$ the parameterization and its mathematical background are presented. In $\S 3$ some theoretical line profiles are calculated, and it is demonstrated that they are well fitted by the proposed parameterization. In $\S 4$ the parameterization is implemented in the Fourier fitting method. In $\S 5$ the new method is applied to long-slit spectra of the galaxies IC 1459, NGC 1374, and NGC 4278. Conclusions are presented in $\S 6$. Auxiliary formulae and results are presented in two appendices.

\section{THE PARAMETERIZATION OF THE LINE PROFILE}

In $\S 2.2$ a new parameterization for the line profiles of elliptical galaxies is presented. This parameterization is discussed in the context of the Fourier fitting method described in detail by FIHa. Note, however, that the parameterization can be used equally well in the context of any of the other methods mentioned in $\S 1$.

\subsection{The Fourier Fitting Method}

The observed galaxy spectrum $\mathscr{G}(\lambda)$ and observed template spectrum $\mathscr{S}(\lambda)$ are rebinned linearly in the quantity $x \equiv \ln \lambda($ a relative velocity $\Delta v$ implies a Doppler shift $\Delta x=\Delta v / c$ ). A polynomial fit is made to the continuum of each spectrum. The continuum fit is subtracted, and the spectra are divided by the average continuum level. It is then assumed that the logarithmically rebinned, continuum-subtracted, normalized spectra $G(x)$ and $S(x)$ satisfy $G(x)=B(x) \circ S(x)$, where $\circ$ denotes convolution. The function $B(x)$ corresponds to the line profile (i.e., the line-of-sight velocity distribution) $\mathscr{L}(v)$, according to $\mathscr{L}(v) \equiv B(v / c)$, and is assumed to depend on a finite number of parameters. The parameters are then determined that minimize the quantity

$$
\chi^{2}=\int[G(x)-B \circ S(x)]^{2} d x .
$$

According to Parseval's theorem, $\chi^{2}$ is equal to the residual $\bar{\chi}^{2}$ in the Fourier domain

$$
\bar{\chi}^{2}=\int[\bar{G}(k)-\bar{B}(k) \bar{S}(k)]^{*}[\bar{G}(k)-\bar{B}(k) \bar{S}(k)] d k,
$$

where $\bar{F}(k)$ denotes the Fourier transform of a function $F(x)$. In the Fourier fitting method $\bar{\chi}^{2}$ is minimized rather than $\chi^{2}$ (the latter is done by $\mathrm{RW}$ ). Methods based on $\chi^{2}$ or $\bar{\chi}^{2}$ minimization have a number of advantages over the traditional Fourier quotient method; e.g., the error analysis is more straightforward (FIHa; RW).

For the sake of mathematical simplicity consider the following idealization:

(i) A perfectly matching template spectrum has been chosen, i.e., $S_{0}(x)=\gamma_{0} S(x), S_{0}(x)$ being the unbroadened galaxy spectrum. The factor $\gamma_{0}$ allows for a difference in line strength between galaxy and template spectrum.

(ii) Information is available at all wavenumbers $k$.

(iii) $|\bar{S}(k)|^{2}=c s t$ for all wavenumbers $k$.

The quantity $\bar{\chi}^{2}$ is then proportional to

$$
\chi_{\mathrm{id}}^{2} \equiv \int_{-\infty}^{\infty}\left[\gamma_{0} \mathscr{L}_{0}(v)-\mathscr{L}(v)\right]^{2} d v,
$$

where $\mathscr{L}_{0}(v)$ is the true normalized distribution of stars over line-of-sight velocities; i.e., by minimizing $\bar{\chi}^{2}$ one is merely least-squares fitting a parameterized function to the true line profile.

In practice the above assumptions are never satisfied. There is always template mismatching. Instrumental broadening and noise limit the accuracy of the information at high wavenumbers. Continuum subtraction limits the accuracy of the information at low wavenumbers. Criterion (iii) is strictly satisfied only for spectra with one single absorption line and negligible intrinsic and instrumental broadening. These points will be addressed in detail in $\S 4$. For the moment let us restrict ourselves to the idealized situation described by equation (2). 


\subsection{The Line Profile as a Sum of Orthogonal Functions}

The usual approach is to assume the line profile to be a Gaussian with free parameters line strength $\gamma$, mean radial velocity $V$, and velocity dispersion $\sigma$,

$$
\mathscr{L}(v)=\gamma \alpha(w) / \sigma, \quad w \equiv(v-V) / \sigma,
$$

where the function $\alpha(y)$ is the standard Gaussian

$$
\alpha(y)=\frac{1}{\sqrt{2 \pi}} e^{-(1 / 2) y^{2}} .
$$

To determine deviations of the line profile from a Gaussian a more general parameterization must be used. To obtain parameters that are only weakly correlated we expand the line profile as a sum of orthogonal functions. Motivated by the fact that Gaussians provide good low-order approximations to most realistic line profiles, we thus consider the Gauss-Hermite series

$$
\mathscr{L}(v)=[\gamma \alpha(w) / \sigma] \sum_{j=0}^{N} h_{j} H_{j}(w), \quad w \equiv(v-V) / \sigma,
$$

where the $h_{l}$ are free parameters, and the functions $H_{l}(y)$ are the Hermite polynomials, defined in Appendix A, that satisfy

$$
\int_{-\infty}^{\infty} H_{m}(y) H_{n}(y) \alpha^{2}(y) d y=\frac{1}{2 \sqrt{\pi}} \delta_{m n} .
$$

Each $H_{l}(y)$ is a polynomial of degree $l$ in $y$. The polynomials $H_{2 m+1}(y)$ of odd degree are antisymmetric in $y$, and therefore the odd coefficients $h_{2 m+1}$ characterize antisymmetric deviations of the line profile from a Gaussian. Similarly the even coefficients characterize symmetric deviations. The quantity $\chi_{\mathbf{i d}}^{2}$ is minimized for

$$
h_{l}^{[\gamma, V, \sigma]}=(2 \sqrt{\pi}) \frac{\gamma_{0}}{\gamma} \int_{-\infty}^{\infty} \mathscr{L}_{0}(v) \alpha(w) H_{l}(w) d v, \quad(l=0, \ldots, N) .
$$

The superscript indicates that this holds for any fixed $(\gamma, V, \sigma)$.

A theorem by Myller-Lebedeff (1908) states that the series equation (5) with $N=\infty$ and the $h_{l}$ as in equation (7) converges to $\gamma_{0} \mathscr{L}_{0}(v)$, for any $V$ and nonzero $\gamma$ and $\sigma$, provided that $\mathscr{L}_{0}(v)$, as well as its first and second derivative, are finite and continuous, and provided that $\lim _{v \rightarrow \pm \infty} v^{3} \mathscr{L}_{0}(v)=0$. These conditions are satisfied for realistic line profiles.

For any choice of $(\gamma, V, \sigma)$ there is thus a Gauss-Hermite series of the form equation (5) that fits the data. This holds even for choices that are far from the true line strength, mean radial velocity, and velocity dispersion. However, to obtain a series that converges rapidly, so that the line profile is already well described by its low-order terms, one has to make sensible choices $(\gamma, V$, $\sigma$ ). It seems natural to choose them such that the lowest order term of the series approximates the line profile as good as possible. This implies setting the parameters $(\gamma, V, \sigma)$ of the Gauss-Hermite expansion equal to the values $(\hat{\gamma}, \hat{V}, \hat{\sigma})$ that define the best Gaussian fit to the line profile. These quantities have the further advantage of being easy to obtain for real data. For this choice it follows from equation (7) (using the definition of the best Gaussian fit) that the corresponding $h_{l}$ are equal to

$$
\begin{aligned}
& \hat{h}_{0}=1, \quad \hat{h}_{1}=0, \quad \hat{h}_{2}=0 . \\
& \hat{h}_{l}=(2 \sqrt{\pi}) \frac{\gamma_{0}}{\hat{\gamma}} \int_{-\infty}^{\infty} \mathscr{L}_{0}(v) \alpha(\hat{w}) H_{l}(\hat{w}) d v, \quad \hat{w} \equiv(v-\hat{V}) / \hat{\sigma}, \quad(l \geq 3) .
\end{aligned}
$$

In practice we find it more convenient to fix $\left(h_{0}, h_{1}, h_{2}\right)$ in equation $(5)$ to $(1,0,0)$, rather than to fix the parameters $(\gamma, V, \sigma)$ to the values corresponding to the best Gaussian fit. Thus functions of the form

$$
\mathscr{L}(v)=[\gamma \alpha(w) / \sigma]\left\{1+\sum_{j=3}^{N} h_{j} H_{j}(w)\right\}, \quad w \equiv(v-V) / \sigma
$$

are considered, and a simultaneous $N+1$ parameter fit is done to find the values $\left(\gamma, V, \sigma, h_{3}, \ldots, h_{N}\right)$ that minimize $\bar{\chi}^{2}$. Henceforth the symbol $h_{l}(l \geq 3)$ will denote quantities obtained in this way. In practice we restrict ourselves to the case $N=4$, but extension to higher orders is straightforward. The bottom panels of Figure 1 demonstrate the effect of nonzero $h_{3}$ and $h_{4}$ on the shape of $\mathscr{L}(v)$. Notice that the wings of $\mathscr{L}(v)$ can be negative, similar to what can happen if a positive function is approximated by the first few terms of its Fourier series. Even though negative wings are unphysical this is no great disadvantage of our method. We wish to extract from real data a number of well-defined parameters (the $\left.h_{l}\right)$, which is independent of how one wishes to physically interpret the fitting function equation (9).

In general the solution $\left(\gamma, V, \sigma, h_{3}, \ldots, h_{N}\right)$ of the simultaneous $N+1$ parameter fit of a function equation (9) to $\gamma_{0} \mathscr{L}_{0}(v)$ is not equal to $\left(\hat{\gamma}, \hat{V}, \hat{\sigma}, \hat{h}_{3}, \ldots, \hat{h}_{N}\right)$. Let $\gamma=\hat{\gamma}(1+\delta \gamma), V=\hat{V}+\hat{\sigma} \delta V, \sigma=\hat{\sigma}(1+\delta \sigma)$, and $h_{l}=\hat{h}_{l}+\delta h_{l}(l=3, \ldots, N)$. The theory of linearized least-squares fits (e.g., Press et al. 1986, § 14) yields that, to lowest order, $\chi_{\text {id }}^{2}$ is minimized for

$$
\begin{aligned}
\delta \gamma & =\frac{1}{2} \delta \sigma \\
\delta V & =\sqrt{2(N+1)} \hat{h}_{N} \hat{h}_{N+1}, \\
\delta \sigma & =\sqrt{N(N+1)} \hat{h}_{N-1} \hat{h}_{N+1}+\sqrt{(N+1)(N+2)} \hat{h}_{N} \hat{h}_{N+2}, \\
\delta h_{l} & =\frac{1}{2} \delta V\left[-\sqrt{2 l} \hat{h}_{l-1}+\sqrt{2(l+1)} \hat{h}_{l+1}\right]+\frac{1}{2} \delta \sigma\left[-\sqrt{l(l-1)} \hat{h}_{l-2}+\sqrt{(l+1)(l+2)} \hat{h}_{l+2}\right], \quad(l=3, \ldots, N),
\end{aligned}
$$



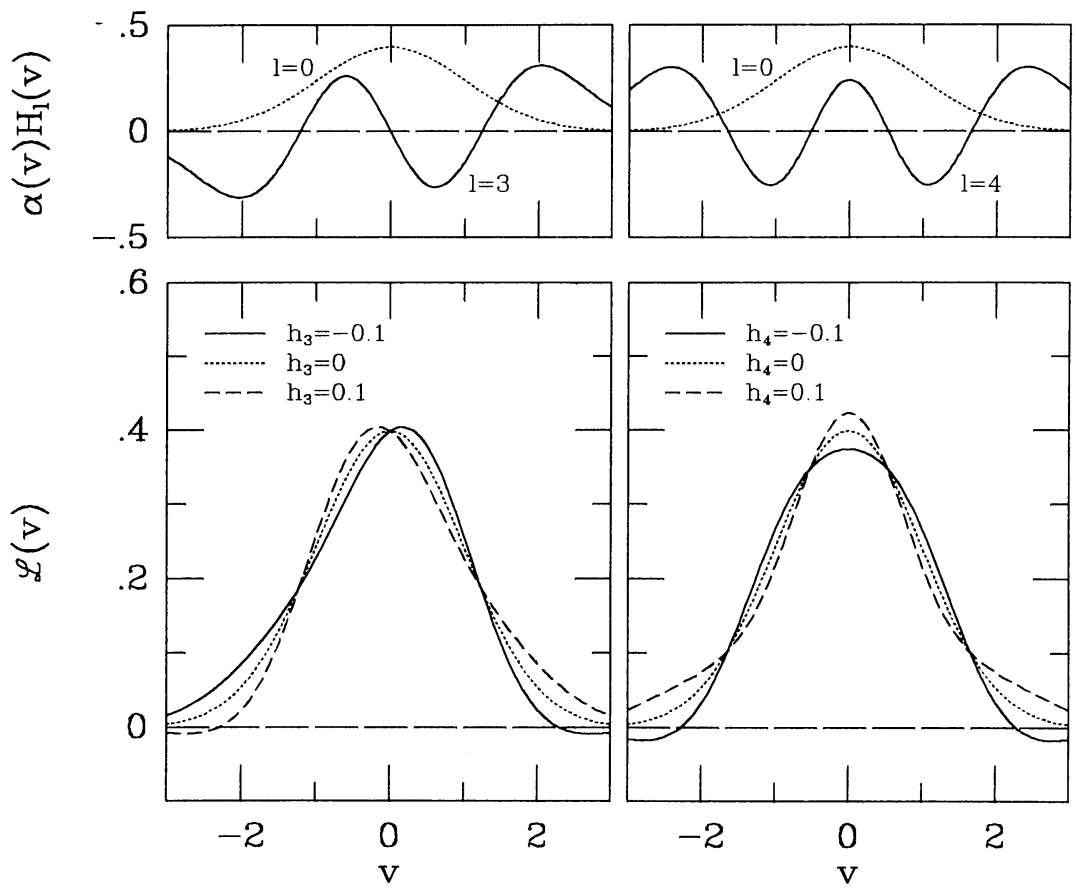

Fig. 1.-(Top panels) Lowest order members $(l=3,4)$ of the orthogonal set of basis functions $\left\{\alpha(v) H_{l}(v)\right\}_{l=3, \ldots, \infty}$ that is used in the present paper to describe deviations of the line profiles of elliptical galaxies from their best-fitting Gaussian. The standard Gaussian $\alpha(v)$ is drawn as a dotted curve. (Bottom panels) The function $\mathscr{L}(v) \equiv \alpha(v)\left[1+h_{3} H_{3}(v)+h_{4} H_{4}(v)\right]$ that is used to parameterize the line profiles, for several combinations of $h_{3}$ and $h_{4}$. The curves in the left panel have $h_{4}=0$ and demonstrate the effect of nonzero $h_{3}$. The peak of $\mathscr{L}(y)$ occurs at negative $y$ for $h_{3}>0$, and vice versa. The curves in the right panel have $h_{3}=0$, and demonstrate the effect of nonzero $h_{4}$. The function $\mathscr{L}(y)$ is more centrally peaked than a Gaussian for $h_{4}>0$, and more flat-topped than a Gaussian for $h_{4}<0$.

where the $\hat{h}_{j}$ are defined by equation (8) for all $j=0, \ldots, \infty$. The quantities $\delta \gamma, \delta V$, and $\delta \sigma$ are typically of the order of one or two percent. The $\delta h_{l}$ are only of order $\hat{h}_{j} \delta q(j \in\{l-2, l-1, l+1, l+2\}, q \in\{V, \sigma\})$. For most practical purposes these quantities are thus negligible. Ordering the parameters as $\left(\gamma, V, \sigma, h_{3}, \ldots, h_{N}\right)$, the correlation matrix of the simultaneous $N+1$ parameter fit is, for $N=4$ and to lowest order in the $\hat{h}_{l}(l \geq 3)$,

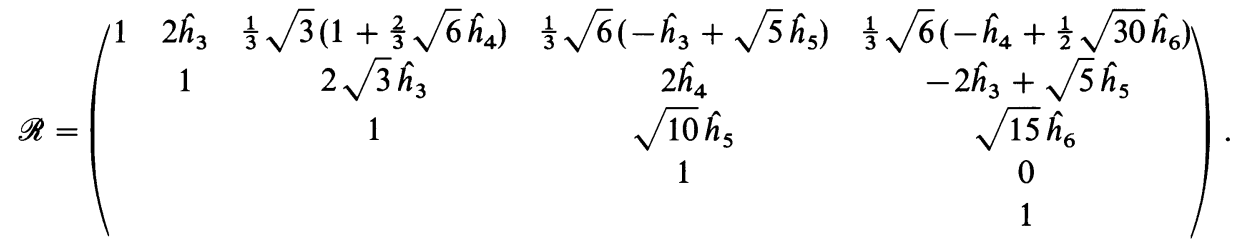

The only zeroth-order correlation is that between $\gamma$ and $\sigma$, which is a known property of fitting Gaussians (Larsen et al. 1983). All other correlations are at least of order $\hat{h}_{l}(l \in\{3, \ldots, 6\})$, and thus generally small for realistic line profiles (cf. $\S 3$ and 4$)$. This constitutes a major advantage of the present parameterization over most alternatives, such as, e.g., a decomposition of the line profile into the sum of two Gaussians.

\subsection{The rms Deviation from the Best-Fitting Gaussian}

We define the rms deviation $\mathscr{D}$ of the line profile $\gamma_{0} \mathscr{L}_{0}(v)$ from its best-fitting Gaussian $\hat{\gamma} \alpha(\hat{w}) / \hat{\sigma}$ by the relation

$$
\mathscr{D}^{2} \equiv \int_{-\infty}^{\infty}\left[\gamma_{0} \mathscr{L}_{0}(v)-\hat{\gamma} \alpha(\hat{w}) / \hat{\sigma}\right]^{2} d v / \int_{-\infty}^{\infty}[\hat{\gamma} \alpha(\hat{w}) / \hat{\sigma}]^{2} d v .
$$

It follows from Myller-Lebedeff's convergence theorem and equation (6) that

$$
\sum_{j=0}^{\infty} \hat{h}_{j}^{2}=2 \sqrt{\pi} \hat{\sigma}\left(\frac{\gamma_{0}}{\hat{\gamma}}\right)^{2} \int_{-\infty}^{\infty}\left[\mathscr{L}_{0}(v)\right]^{2} d v .
$$

The rms deviation $\mathscr{D}$ is thus related to the $\hat{h}_{l}(l \geq 3)$ according to

$$
\mathscr{D}=\left(\sum_{j=3}^{\infty} \hat{h}_{j}^{2}\right)^{1 / 2} \text {. }
$$




\subsection{Relation to the Moments of the Line Profile}

The $k$ th-moment of the normalized line profile $\mathscr{L}_{0}(v)$ is

$$
\mu_{k} \equiv \int_{-\infty}^{\infty} \mathscr{L}_{0}(v)\left(v-V_{0}\right)^{k} d v
$$

The mean velocity is $V_{0} \equiv \int_{-\infty}^{\infty} \mathscr{L}_{0}(v) v d v$ and the velocity dispersion is $\sigma_{0} \equiv \sqrt{\mu_{2}}$. The coefficient of skewness $\xi_{1} \equiv \mu_{3} / \mu_{2}^{3 / 2}$ and the coefficient of kurtosis $\xi_{2} \equiv \mu_{4} / \mu_{2}^{2}$ measure deviations of the line profile from a Gaussian (for which $\xi_{1}=0$ and $\xi_{2}=3$ ). Note that the $h_{l}$ are less susceptible to the wings of the line profile (which are ill-constrained by the observations) than are the $\mu_{l}$, due to the factor $\alpha(w)$ in equation (7).

Usually the true line strength $\gamma_{0}$, mean radial velocity $V_{0}$, and velocity dispersion $\sigma_{0}$ are estimated by fitting a Gaussian to the data. However, if the true line profile is not a Gaussian, and if information $\left(h_{3}, h_{4}\right)$ is available on the deviations of the line profile from a Gaussian, it is possible to obtain better estimates. One should not simply calculate the moments of the fitting function $\mathscr{L}(v)$ defined by equation (9). This yields unsatisfactory results because the wings of $\mathscr{L}(v)$ can be slightly negative (see Fig. 1) which influences the moments disproportionately. Instead we consider the physically more meaningful function.

$$
\tilde{\mathscr{L}}(v) \equiv \max [\mathscr{L}(v), 0] .
$$

Given the parameters $\left(\gamma, V, \sigma, h_{3}, h_{4}\right)$ returned by the Fourier fitting program, estimates $\left(\tilde{\gamma}, \tilde{V}, \tilde{\sigma}, \tilde{\xi}_{1}, \tilde{\xi}_{2}\right)$ of the true values of $\left(\gamma_{0}, V_{0}\right.$, $\left.\sigma_{0}, \xi_{1}, \xi_{2}\right)$ are calculated from

$$
\tilde{\gamma} \equiv \int_{-\infty}^{\infty} \tilde{\mathscr{L}}(v) d v, \quad \tilde{V} \equiv \int_{-\infty}^{\infty} v[\tilde{\mathscr{L}}(v) / \tilde{\gamma}] d v, \quad \tilde{\sigma}^{2} \equiv \int_{-\infty}^{\infty}[(v-\tilde{V})]^{2}[\tilde{\mathscr{L}}(v) / \tilde{\gamma}] d v, \quad \tilde{\xi}_{i} \equiv \int_{-\infty}^{\infty}[(v-\tilde{V}) / \tilde{\sigma}]^{2+i}[\tilde{\mathscr{L}}(v) / \tilde{\gamma}] d v .
$$

The lowest order in $h_{3}$ and $h_{4}$

$$
\tilde{\gamma}=\gamma\left(1+\frac{1}{4} \sqrt{6} h_{4}\right), \quad \tilde{V} \approx V+\sqrt{3} \sigma h_{3}, \quad \tilde{\sigma} \approx \sigma\left(1+\sqrt{6} h_{4}\right), \quad \tilde{\xi}_{1} \approx 4 \sqrt{3} h_{3}, \quad \tilde{\xi}_{2} \approx 3+8 \sqrt{6} h_{4} .
$$

In practice these quantities are calculated numerically. ${ }^{2}$ In $\S 3$ it is demonstrated that for realistic line profiles $(\tilde{\gamma}, \tilde{V}, \tilde{\sigma})$ are better estimates of the true moments $\left(\gamma_{0}, V_{0}, \sigma_{0}\right)$ than are the parameters $(\hat{\gamma}, \hat{V}, \hat{\sigma})$ that define the best-fitting Gaussian.

\section{THEORETICAL LINE PROFILES FOR THE OUTER PARTS OF ELLIPTICAL GALAXIES}

This paragraph addresses the question whether realistic line profiles are well fitted by the parameterization presented in $\S 2$. We have calculated some line profiles for the outer parts of spherically symmetric, anisotropic models for elliptical galaxies. Let the relative potential of the system be $\Psi \equiv-\Phi$, and the relative energy of a star be $\mathscr{E} \equiv \Psi(r)-\frac{1}{2} v^{2}$ (cf. Binney \& Tremaine 1987). The relative potential is either assumed to be Keplerian, $\Psi=G M / r, M$ being the total mass of the system, or logarithmic, $\Psi=-V_{c}^{2} \ln r$. These potentials roughly correspond to the outer parts of the system, without or with a dark halo, respectively. First, nonrotating models are considered in which the phase space distribution function of the stars (assumed to behave as test particles in the given potential) is

$$
f(\mathscr{E}, L)=L^{-2 \beta} f_{0}(\mathscr{E}),
$$

where $L \equiv r\left(v_{\phi}^{2}+v_{\theta}^{2}\right)^{1 / 2}$ is the angular momentum per unit mass, and $\beta<1$ is a constant. The mass-density of the stars is assumed to follow a power law

$$
\rho(r) \equiv \int L^{-2 \beta} f_{0}(\mathscr{E}) d^{3} v=C r^{-t},
$$

where $C$ is a constant. This equation defines $f_{0}(\mathscr{E})$, for given potential $\Psi$. Distribution functions of the type equation (19) have the property that $1-\sigma_{\theta}^{2} / \sigma_{r}^{2}=\beta$ everywhere (e.g., Hénon 1973). For $\beta=0$ the system is isotropic, in the limit $\beta \rightarrow 1$ the system is built of radial orbits only, and in the limit $\beta \rightarrow-\infty$ the system is built of circular orbits only. For this model all the moments of the line profile can be calculated analytically (Appendix B, § B1), and from these the line profile can be reconstructed (Appendix B, $\S$ B2). Figures $2 a$ and $2 b$, show the line profiles for $t=4$ and various values of $\beta$. The line profile for $\beta \rightarrow-\infty$ can be significantly double-peaked. The line profiles become more centrally peaked with increasing $\beta$. The line profile for $\beta \rightarrow 1$ diverges logarithmically for $v \rightarrow 0$.

Simple rotating models with the same mass density are obtained by reversing stellar velocities such that in every point a fraction $\zeta$ of the stars has positive $v_{\phi}$ and a fraction $1-\zeta$ has negative $v_{\phi}$. For the logarithmic potential and $\beta=0$ the resulting line profiles are particularly easy to calculate (Appendix B, $\S$ B3; see Toomre 1982 and Evans 1993 for related calculations). In Figure 2c the line profiles are plotted for $t=4$ and various values of $\zeta$, for the case that the system is viewed from within the equatorial plane.

Each of the line profiles in Figure 2 was decomposed into a Gauss-Hermite series. A Gaussian was fitted to obtain the parameters $(\hat{\gamma}, \hat{V}, \hat{\sigma})$. The parameters $\hat{h}_{3}$ and $\hat{h}_{4}$ were calculated from equation $(8)$. The rms deviation $\mathscr{D}$ of the line profile from its best-fitting Gaussian was calculated from equations (13) and (14). Improved estimates $(\tilde{\gamma}, \widetilde{V}, \tilde{\sigma})$ of the true moments of the line profile $\left(\gamma_{0}, V_{0}, \sigma_{0}\right)$

\footnotetext{
${ }^{2}$ Formal errors $\left(\Delta \tilde{\gamma}, \Delta \tilde{V}, \Delta \tilde{\sigma}, \Delta \tilde{\xi}_{1}, \Delta \tilde{\xi}_{2}\right)$ are calculated from the covariance matrix $\Sigma$ returned by the Fourier fitting program. From the standard theory of first-order error analysis it follows that the error in a scalar function $f(y), y$ being a vector with elements $y_{i}$, is $\Delta f=\left(f^{\prime T} \Sigma f^{\prime}\right)^{1 / 2}$, where $f^{\prime}$ is the column vector with elements $\left(\partial f / \partial y_{i}\right)$ and $\Sigma$ is the covariance matrix of the $y_{i}$
} 


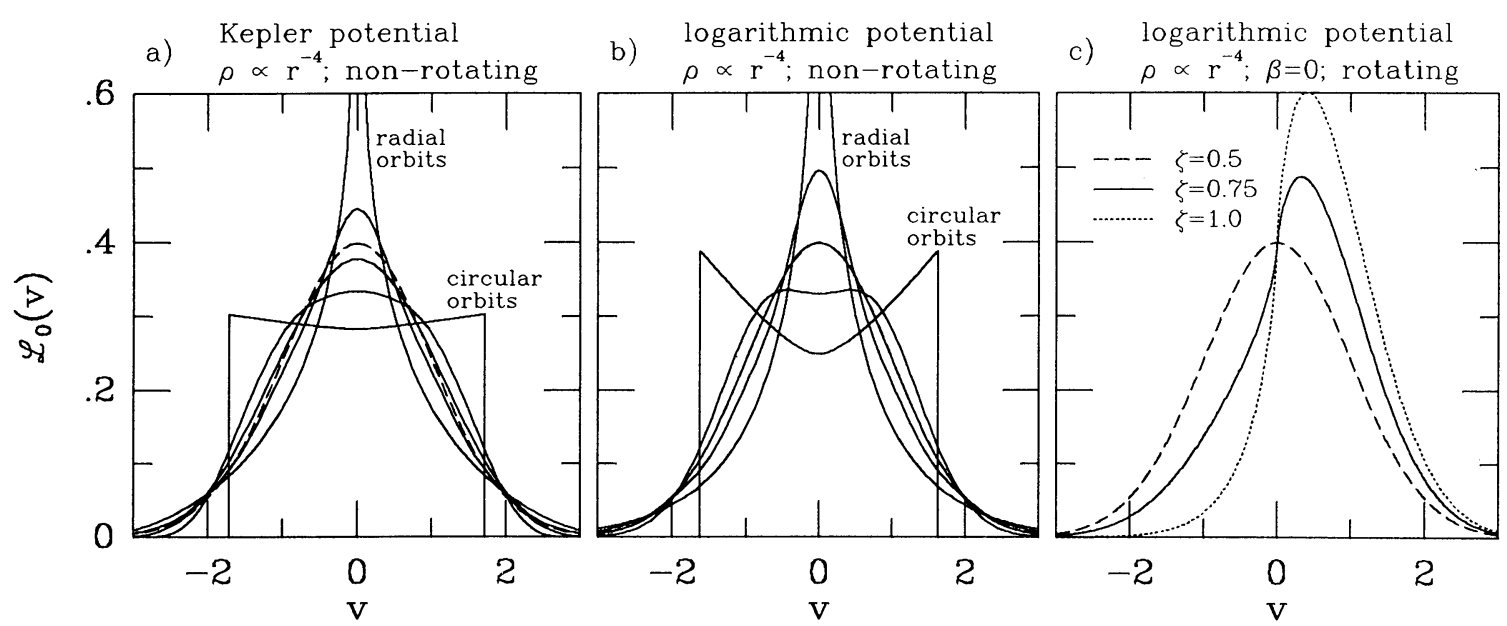

FIG. 2.-Normalized line profiles $\mathscr{L}_{0}(v)$ of models for the outer parts of spherical galaxies in which the stellar mass density is $\rho \propto r^{-4}$. In panels $(a)$ and $(b)$ the models are nonrotating, the potential is either $(a)$ Keplerian or $(b)$ logarithmic, and the (constant) velocity dispersion anisotropy is $\beta=-\infty$ (circular orbit model), $-1,0$ (isotropic model), $\frac{1}{2}$, or 1 (radial orbit model). The unit of velocity is the velocity dispersion $\sigma_{0}$, which is thus different for each curve. In panel $(c)$ the potential is logarithmic, $\beta=0$, and the models are rotating: in every point a fraction $\zeta$ of the stars has positive $v_{\phi}$ and a fraction $1-\zeta$ has negative $v_{\phi}$. The line of sight is assumed to lie in the equatorial plane. The unit of velocity is the rms projected line-of-sight velocity, which is $V_{c} / 2$. For $\zeta=0.75$ the line profile has $V_{0} / \sigma_{0}=0.36$; for $\zeta=1$, $V_{0} / \sigma_{0}=0.92$. In all panels the standard Gaussian is indicated by the dashed curve. It coincides with the isotropic model in panel $(b)$.

were calculated as described in $\S 2.4$. Table 1 lists the results. Figure 3 shows for three specific line profiles the best Gaussian fit and the fit if $\hat{h}_{3}$ and $\hat{h}_{4}$ are included. We conclude the following:

(i) For the most extreme velocity dispersion anisotropies $(\beta \rightarrow-\infty$ or $\beta \rightarrow 1)$ the line profiles do not resemble a Gaussian at all. For more modest (and more plausible) anisotropies, most of the total rms deviation from the best-fitting Gaussian is accounted for by $\hat{h}_{3}$ and $\hat{h}_{4}$. The parameterization equation (9) with $N=4$ thus provides a good fit to realistic line profiles.

(ii) The estimates $(\hat{\gamma}, \hat{V}, \hat{\sigma})$ of the true line strength $\gamma_{0}$, mean radial velocity $V_{0}$, and velocity dispersion $\sigma_{0}$, that are obtained by fitting a Gaussian to the line profile, can be significantly in error. For example, for nonrotating models with modest anisotropies (such as $\beta=-1$ or $\beta=\frac{1}{2}$ ), the estimate of the line strength is in error by $\sim 3 \%$, and that of the velocity dispersion is in error by $\sim 10 \%$. For radially anisotropic models $\hat{\gamma}$ and $\hat{\sigma}$ are underestimates of $\gamma_{0}$ and $\sigma_{0}$, whereas for tangentially anisotropic models they are overestimates. For the rotating models discussed here the estimate of the rotation velocity can be in error by $10 \%$ or more.

(iii) The quantities $(\tilde{\gamma}, \tilde{V}, \tilde{\sigma})$ discussed in $\S 2.4$ are generally much better estimates of $\left(\gamma_{0}, V_{0}, \sigma_{0}\right)$ than are $(\hat{\gamma}, \hat{V}, \hat{\sigma})$. The quantities $\tilde{\xi}_{1}$ and $\tilde{\xi}_{2}$, on the other hand, were found not to be particularly accurate estimates of the true coefficients of skewness and kurtosis. These quantities are thus not used any further.

TABLE $1^{\mathrm{a}}$

Characteristics of the Line Profiles in Figure 2

\begin{tabular}{|c|c|c|c|c|c|c|c|c|c|c|}
\hline$\beta$ & $\zeta$ & $\hat{\gamma} / \gamma_{0}$ & $\hat{V} / V_{0}$ & $\hat{\sigma} / \sigma_{0}$ & $\hat{h}_{3}$ & $\hat{h}_{4}$ & $\mathscr{D}$ & $\tilde{\gamma} / \gamma_{0}$ & $\tilde{V} / V_{0}$ & $\tilde{\sigma} / \sigma_{0}$ \\
\hline \multicolumn{11}{|c|}{ Kepler Potential; $\rho \propto r^{-4} ;$ Nonrotating System } \\
\hline$-\infty$ & $\ldots$ & 1.063 & $\ldots$ & 1.243 & $\ldots$ & -0.194 & 0.369 & 1.041 & $\ldots$ & 1.105 \\
\hline$-1 \ldots \ldots \ldots$ & $\ldots$ & 1.037 & $\cdots$ & 1.139 & $\cdots$ & -0.094 & 0.108 & 1.011 & $\cdots$ & 1.028 \\
\hline $0 \ldots \ldots \ldots \ldots$ & $\cdots$ & 1.015 & $\cdots$ & 1.058 & $\cdots$ & -0.027 & 0.028 & 1.001 & $\cdots$ & 1.003 \\
\hline $0.5 \ldots \ldots \ldots \ldots$ & $\ldots$ & 0.984 & $\ldots$ & 0.956 & $\ldots$ & 0.044 & 0.062 & 1.011 & $\ldots$ & 1.050 \\
\hline $1 \ldots \ldots \ldots \ldots$ & $\cdots$ & 0.876 & $\cdots$ & 0.715 & $\cdots$ & 0.204 & 0.279 & 0.986 & $\cdots$ & 0.982 \\
\hline \multicolumn{11}{|c|}{ Logarithmic Potential; $\rho \propto r^{-4}$; Nonrotating System } \\
\hline$-\infty$ & $\ldots$ & 1.071 & $\ldots$ & 1.271 & $\ldots$ & -0.228 & 0.478 & 1.052 & $\ldots$ & 1.130 \\
\hline$-1 \ldots \ldots \ldots$ & $\ldots$ & 1.031 & $\ldots$ & 1.112 & $\ldots$ & -0.083 & 0.105 & 1.006 & $\cdots$ & 1.008 \\
\hline $0 \ldots \ldots \ldots \ldots$ & $\ldots$ & 1.000 & $\ldots$ & 1.000 & $\ldots$ & 0.000 & 0.000 & 1.000 & $\ldots$ & 1.000 \\
\hline $0.5 \ldots \ldots \ldots \ldots$ & $\ldots$ & 0.956 & $\ldots$ & 0.858 & $\ldots$ & 0.085 & 0.099 & 1.006 & $\ldots$ & 1.014 \\
\hline $1 \ldots \ldots \ldots \ldots$ & $\ldots$ & 0.791 & $\ldots$ & 0.458 & $\ldots$ & 0.253 & 0.386 & 0.913 & $\ldots$ & 0.659 \\
\hline \multicolumn{11}{|c|}{ Logarithmic Potential; $\rho \propto r^{-4} ;$ Rotating System } \\
\hline$\ldots \ldots$ & 0.75 & 0.970 & 1.195 & 0.906 & -0.040 & 0.064 & 0.090 & 1.007 & 1.015 & 1.023 \\
\hline $0 \ldots \ldots \ldots \ldots$ & 1.0 & 0.971 & 0.900 & 0.897 & 0.086 & 0.039 & 0.117 & 0.987 & 1.016 & 0.935 \\
\hline
\end{tabular}

a The parameters $\beta$ and $\zeta$ define the velocity structure of the model, as described in the text. The quantities $\left(\gamma_{0}, V_{0}, \sigma_{0}\right)$ are the true line strength, mean radial velocity and velocity dispersion. The quantities $(\hat{\gamma}, \hat{V}, \hat{\sigma})$ are estimates of these quantities obtained by fitting a Gaussian to the line profile. The quantities $\hat{h}_{3}, \hat{h}_{4}$, and $\mathscr{D}$ (eqs. [8, 14]) measure deviations of the line profile from a Gaussian. The quantities $(\tilde{\gamma}, \tilde{V}, \tilde{\sigma})$ are improved estimates of $\left(\gamma_{0}, V_{0}, \sigma_{0}\right)$, obtained as described in $\$ 2.4$. 


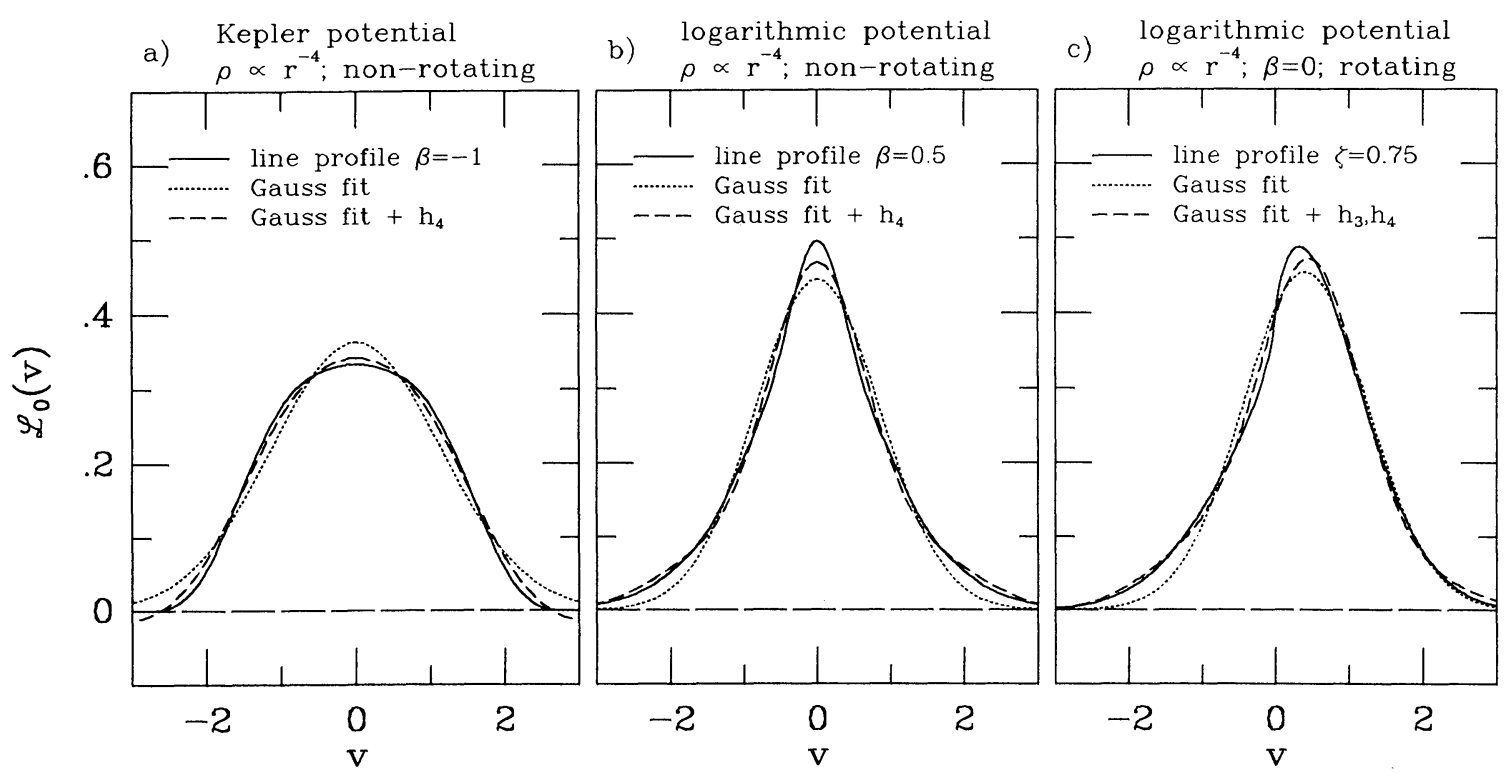

Fig. 3. - Three selected line profiles from Fig. 2, one from each panel. Shown are the best Gaussian fit and the fit if $h_{3}$ and $\hat{h}_{4}$ are included. In all three panels inclusion of $\hat{h}_{3}$ and $\hat{h}_{4}$ decreases the rms deviation between the line profile and the fit from $\sim 10 \%$ to $\sim 5 \%$.

(iv) In the above nonrotating models knowledge of $h_{4}$ directly yields information on the anisotropy of the stars in the outer parts of the galaxy. This anisotropy contains clues on how the galaxy formed. Knowing the anisotropy, the behavior of the velocity dispersion as a function of radius gives constraints on the presence of a dark halo (see also: Gerhard 1991). On the other hand, even if $h_{4}$ could be determined with enough accuracy, far enough out in the galaxy, a number of questions remain to be answered, e.g.: (i) to what extent can the results derived here for the limit of large radii, be used to model data at, say, one effective radius?; and (ii) to what extent do the results for the models presented here, differ from other possible models, in which, e.g., the velocity dispersion anisotropy of the stars changes with radius? These questions are beyond the scope of the present paper. The line profiles calculated here merely serve to demonstrate the usefulness of the parameterization introduced in $\S 2$.

\section{TESTS OF THE METHOD}

The parameterization presented in $\S 2$ was implemented in the Fourier fitting method. The linearized least-squares fitting algorithm described in Appendix A of FIHa is used to determine the $\left(\gamma, V, \sigma, h_{3}, h_{4}\right)$ that minimize $\bar{\chi}^{2}$ (eq. [1b]), for $\mathscr{L}(v)$ as in equation (9). This algorithm returns the best-fitting parameters, their formal errors, and the covariance matrix of the fit.

The determination of line profile shapes requires spectra with high signal-to-noise (RW). For the observations in the $\mathbf{M g} b$ triplet region presented in $\S 5$, we found that a S/N of at least 50 per $\AA$ was required to obtain useful estimates of $h_{3}$ and $h_{4}$.

The quantity $\bar{\chi}^{2}$ is evaluated over a finite range of wavenumbers $k$. The use of an upper cutoff $k_{U}$ is motivated by the fact that there is no information in the highest wavenumbers, due to the finite spectral resolution of the instrument. For the observations presented in $\S 5$ this resolution was typically between 0.2 and 0.5 times the velocity dispersion of the galaxy (Table 3 ). For these data the results of the Fourier fitting program were insensitive to the choice of $k_{U}$. There is also no great dependence of the results on spectral resolution, as will be demonstrated in $\S 5$.

Low wavenumber information that remains after the continua of galaxy and template spectrum have been subtracted cannot be trusted and is filtered out of the Fourier transformed spectra. An inverted cosine-bell filter is used, i.e., zero from wavenumber zero to some user defined wavenumber $k_{L}$, and then smoothly rising to 1 at $2 k_{L}$. The particular choice for $k_{L}$ is more or less arbitrary within a factor of 2 or so; $k_{L}=(70 \AA)^{-1}$ was adopted. For a Gaussian line profile with dispersion $\sigma_{0}$ in velocity space and corresponding dispersion $\sigma_{k}$ in Fourier space, this implies that no information is available at wavenumbers $k \lesssim 0.5 \sigma_{k}\left(\sigma_{0} / 300 \mathrm{~km}\right.$ $\mathrm{s}^{-1}$ ). Information on the wings and the moments of the line profile is thus missing (the $k$ th moment of the line profile is proportional to the $k$ th derivative of its Fourier transform at wavenumber zero), the more so for larger $k_{L}$ and $\sigma_{0}$. The results of the Fourier fitting program can therefore depend weakly on the choice of $k_{L}$, especially if the parameterization of the line profile does not provide a good fit. To study the dependence of $h_{3}$ and $h_{4}$ on $k_{L}$, simulated spectra were created by convolving an observed stellar spectrum with the line profiles calculated in $\S 3$, setting $\sigma_{0} \approx 300 \mathrm{~km} \mathrm{~s}^{-1}$. The Fourier fitting program was then applied with $k_{L}=(70 \AA)-1$, and the same star as template. The differences between the results for $h_{3}$ and $h_{4}$ and the values listed in Table 1 were small, typically between -0.02 and 0.02 . For real data it was found that $h_{3}$ and $h_{4}$ typically change by an amount between -0.03 and 0.03 , if $k_{L}$ is varied within the plausible range. These changes tend to be the same at all radii along the slit; that is, the typical effect of varying $k_{L}$ is to introduce a small constant offset in the profiles $h_{3}(r)$ and $h_{4}(r), r$ being the distance along the slit.

The discussion in $\S 2$ assumes that integrals can be evaluated over the range $-\infty<v<\infty$, which requires information at all wavenumbers. As a consequence of the fact that this information is not available, the Hermite polynomials cease being strictly orthogonal. The correlations between the errors in the parameters of the fit are in practice therefore slightly larger than given in equation (11). 
VAN DER MAREL \& FRANX

TABLE $2^{\mathrm{a}}$

Results of Tests with Simulated Data

\begin{tabular}{|c|c|c|c|c|c|c|c|}
\hline Type & {$[\mathrm{Fe} / \mathrm{H}]$} & Star & $\left\langle h_{3}\right\rangle$ & $\sigma_{h_{3}}$ & $\left\langle h_{4}\right\rangle$ & $\sigma_{h_{4}}$ & $\left\langle\chi_{\mathrm{red}}^{2}\right\rangle$ \\
\hline G8 V & & HD 51219 & 0.04 & 0.02 & 0.06 & 0.03 & 1.72 \\
\hline G5 IV & $\ldots$ & HD 125968 & 0.03 & 0.02 & 0.04 & 0.03 & 1.75 \\
\hline G8 IV & $\ldots$ & HD 170829 & 0.01 & 0.01 & 0.04 & 0.02 & 1.68 \\
\hline . . & .. & HD 81192 & 0.00 & 0.02 & 0.00 & 0.03 & 1.19 \\
\hline G9 III . & 0.32 & HD 72324 & -0.01 & 0.01 & 0.00 & 0.02 & 1.54 \\
\hline K0 III . & 0.10 & HD 85503 & 0.00 & 0.01 & -0.02 & 0.01 & 1.09 \\
\hline K0 III . & -0.65 & HD 102224 & 0.00 & 0.01 & 0.00 & 0.01 & 1.35 \\
\hline K4 III . & $\ldots$ & HD 93102 & 0.00 & 0.01 & -0.02 & 0.01 & 1.05 \\
\hline M1 III & $\ldots$ & HD 84542 & 0.00 & 0.01 & -0.09 & 0.01 & 7.35 \\
\hline
\end{tabular}

${ }^{\text {a }}$ Results of tests to assess the influence of template mismatching on $h_{3}$ and $h_{4}$, as described in the text.

The process of deriving deviations from a Gaussian line profile from observations is sensitive to mismatch between galaxy and template spectrum (RW). Table 2 describes the results of tests to assess the influence of template mismatching on $h_{3}$ and $h_{4}$. Simulated galaxy spectra were created by convolving observed spectra (spectral resolution $\sigma=60 \mathrm{~km} \mathrm{~s}^{-1}$ ) of stars of different spectral types and metallicities with a Gaussian of width $\sigma_{0}=250 \mathrm{~km} \mathrm{~s}^{-1}$. Of each resulting spectrum 30 copies were made, and to each copy a realization of Poisson noise with S/N of 75 per $\AA$ was added. These spectra were then analyzed with the Fourier fitting program using the K0 III star HD 56224 as template. Listed in Table 2 are for each input stellar spectrum the mean $\left\langle h_{l}\right\rangle$ and dispersion $\sigma_{h_{l}}$ of the 30 output values of $h_{3}$ and $h_{4}$. Also listed is the mean $\left\langle\chi_{\text {red }}^{2}\right\rangle$ of the reduced $\chi^{2}$-values (being defined as the ratio of the $\chi^{2}$ returned by the fit and the $\chi^{2}$ expected for pure Poisson noise and no template mismatch). This quantity is a measure for the amount of template mismatching. The input stars were compiled from the lists of Cayrel de Strobel et al. (1985) and Pickles (1985a). Half of the light from elliptical galaxies at $5500 \AA(\sim 55 \%)$ comes from $\mathrm{G}$ and $\mathrm{K}$ giants (Pickles 1985b). However, other spectral types such as $\mathrm{M}$ giants (few percent), $\mathrm{G}$ subgiants $(\sim 25 \%)$, and $\mathrm{G}$ dwarfs $(\sim 15 \%)$ also contribute. Table 2 thus suggests that typical systematic errors in $h_{3}$ and $h_{4}$ due to template mismatching are $-0.02 \lesssim \Delta h_{3} \lesssim 0.02$ and $-0.03 \lesssim \Delta h_{4} \lesssim 0.03$. The stars that produce the highest systematic errors also have high $\chi_{\text {red }}^{2}$, which is very significant at this $\mathbf{S} / \mathrm{N}$. The worst effects of template mismatching can thus be avoided by selecting the template that produces the lowest $\chi_{\text {red. }}^{2}$. In practice, repeated application of the Fourier fitting program to the same galaxy data using different plausible stellar spectra as template yields the same profiles $h_{3}(r)$ and $h_{4}(r)$, but slightly offset with respect to one another. The absence of a dependence on $r$ is what should be expected in the absence of large stellar population gradients. The offsets are typically between -0.03 and 0.03 , consistent with the results in Table 2 . We have not pursued the approach of RW to use a linear combination of different stellar spectra as template and fit template and line profile to the data simultaneously. This approach has the advantage of improving the fit to the observed galaxy spectrum, but also gives the fitting program the possibility to incorrectly choose a particular spectral mix, to mimic what in reality is a non-Gaussian line profile.

In an equilibrium stellar system, the line profiles at diametrically opposed points are expected to show reflection symmetry. For slits centered on the nucleus, $h_{3}(r)$ should thus be an odd function of $r$, and $h_{4}(r)$ should be an even function of $r$. Any artificial constant offset in the profile $h_{3}(r)$ returned by the Fourier fitting program can thus be determined. For the data discussed in $\S 5$ and plotted in Figure 4, the offsets in $h_{3}(r)$ are small, between -0.02 and 0.02 . Since $h_{4}(r)$ should be an even function of $r$, any artificial constant offset in $h_{4}(r)$ is likely to remain undetected as such. The profiles $h_{3}(r)$ and $h_{4}(r)$ plotted in Figure 4 are (discarding the offsets in $h_{3}$ ), indeed odd and even functions of $r$, respectively. This, together with the small sizes of the offsets in $h_{3}$, provides extra confidence in the validity of these results.

To summarize, profiles $h_{3}(r)$ and $h_{4}(r)$ can be determined from observations with sufficient $\mathrm{S} / \mathrm{N}$. The profile $h_{4}(r)$ can only be determined up to a small constant offset (usually between -0.03 and 0.03 ), due to the filtering required at low wavenumbers and the problems associated with template mismatching. In practice it must always be checked to what extent the results of the Fourier fitting program depend on the choice of the template spectrum. The dependence of the results on the choice of $k_{L}$ can, at least in principle, be corrected for by subjecting model line profiles that one wishes to compare to the data to the same filtering that was applied to the data itself.

\section{APPLICATION TO REAL DATA: IC 1459, NGC 1374, AND NGC 4278}

Long-slit spectra, centered on the $\mathrm{Mg} b$ triplet, were taken of three elliptical galaxies: IC 1459 (E4), NGC 1374 (E0), and NGC 4278 (E1). The data were taken by MF, in collaboration with Garth Illingworth, using the $4 \mathrm{~m}$ telescopes of the CTIO and KPNO. They form part of a more extensive survey on which we intend to report in a future paper. Characteristics of the data are listed in Table 3. The data were wavelength calibrated and reduced using standard IRAF tasks. The results of the Fourier fitting method are plotted in Figures $4 a-4 d$. Spectra were, if necessary, summed along the slit until a $\mathrm{S} / \mathrm{N}$ of at least 75 per pixel were reached. The K0 III star HD 56224 was used as template.

IC 1459 and NGC 4278 have emission lines of [O III] $4959 \AA$, [O III] $5007 \AA$, and [N I] $5200 \AA$. These lines were interpolated over by replacing the galaxy data on and around the emission lines by the best-fit to the galaxy spectrum returned by the Fourier fitting program. This procedure was iterated a few times to make sure that the final results were not influenced by the presence of the emission lines.

IC 1459 has a counter-rotating core (FI; FIHa). It is included here because large deviations from a Gaussian line profile were detected earlier by FI. They found that the line profiles on the major axis are asymmetric, that the asymmetry changes sign upon going from one side of the nucleus to the other, and that the tail of the line profile is away from the direction of rotation (as in Fig. 5 


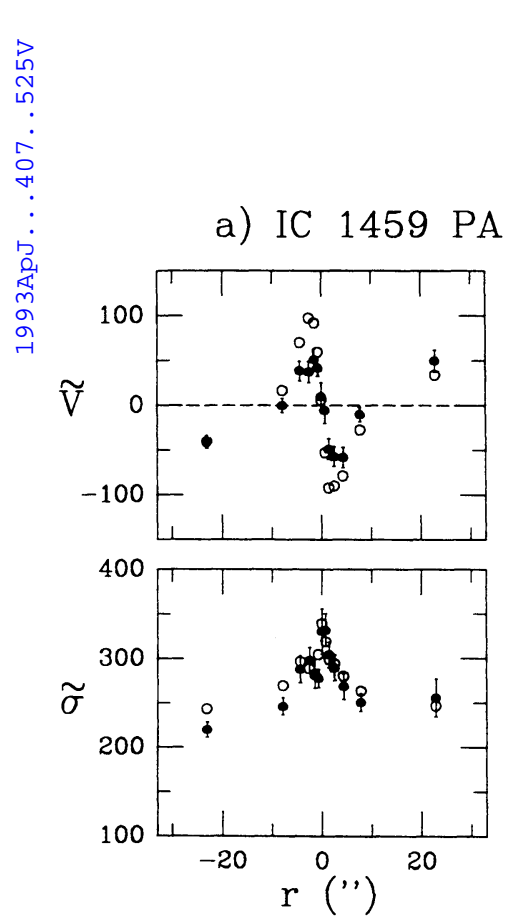

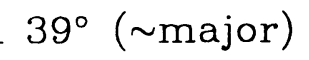

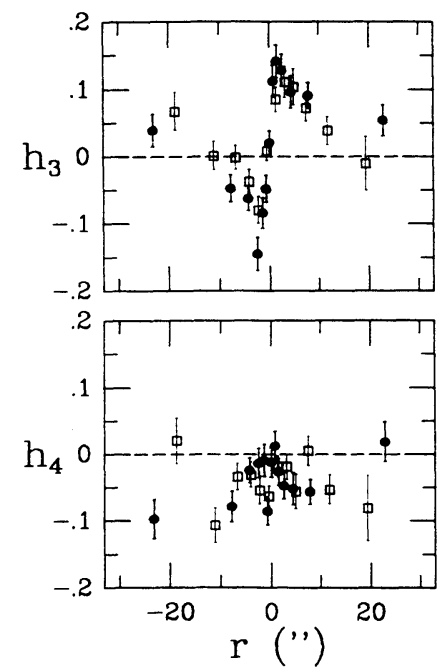

c) NGC $1374 \mathrm{PA} 110^{\circ}$ ( major)
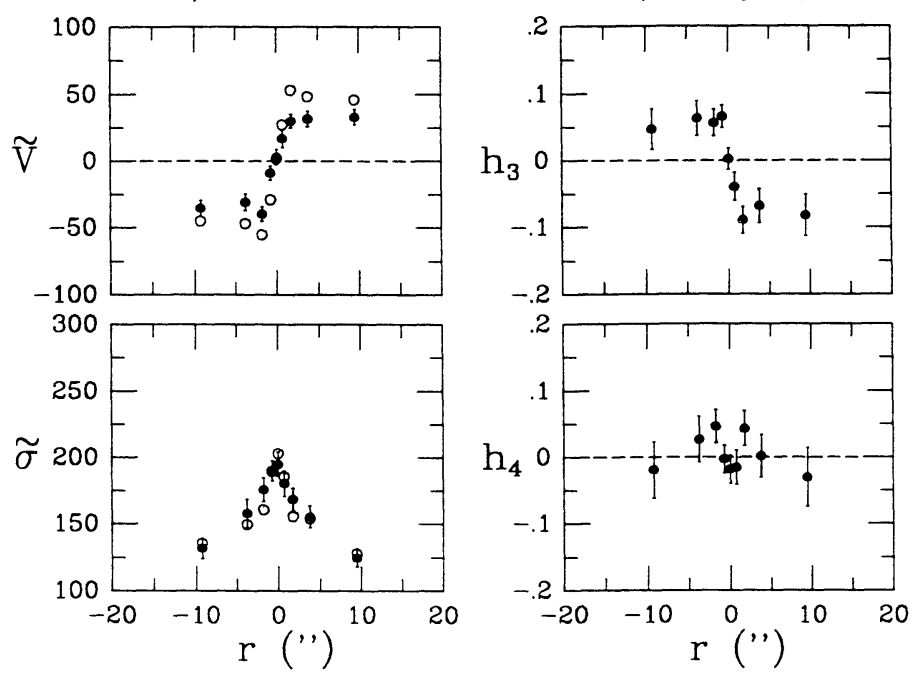
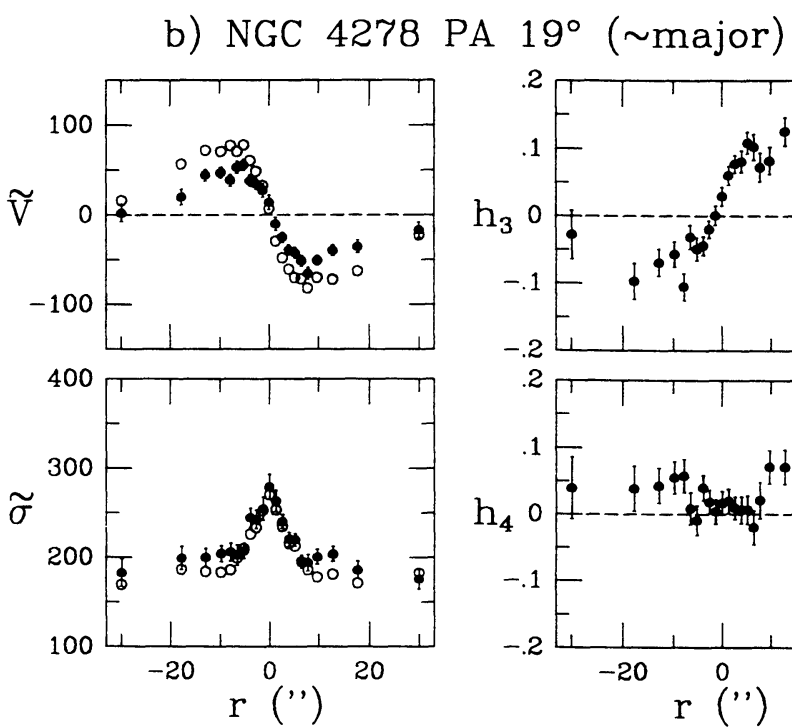

d) NGC $1374 \mathrm{PA} 20^{\circ}$ ( $\sim$ minor)
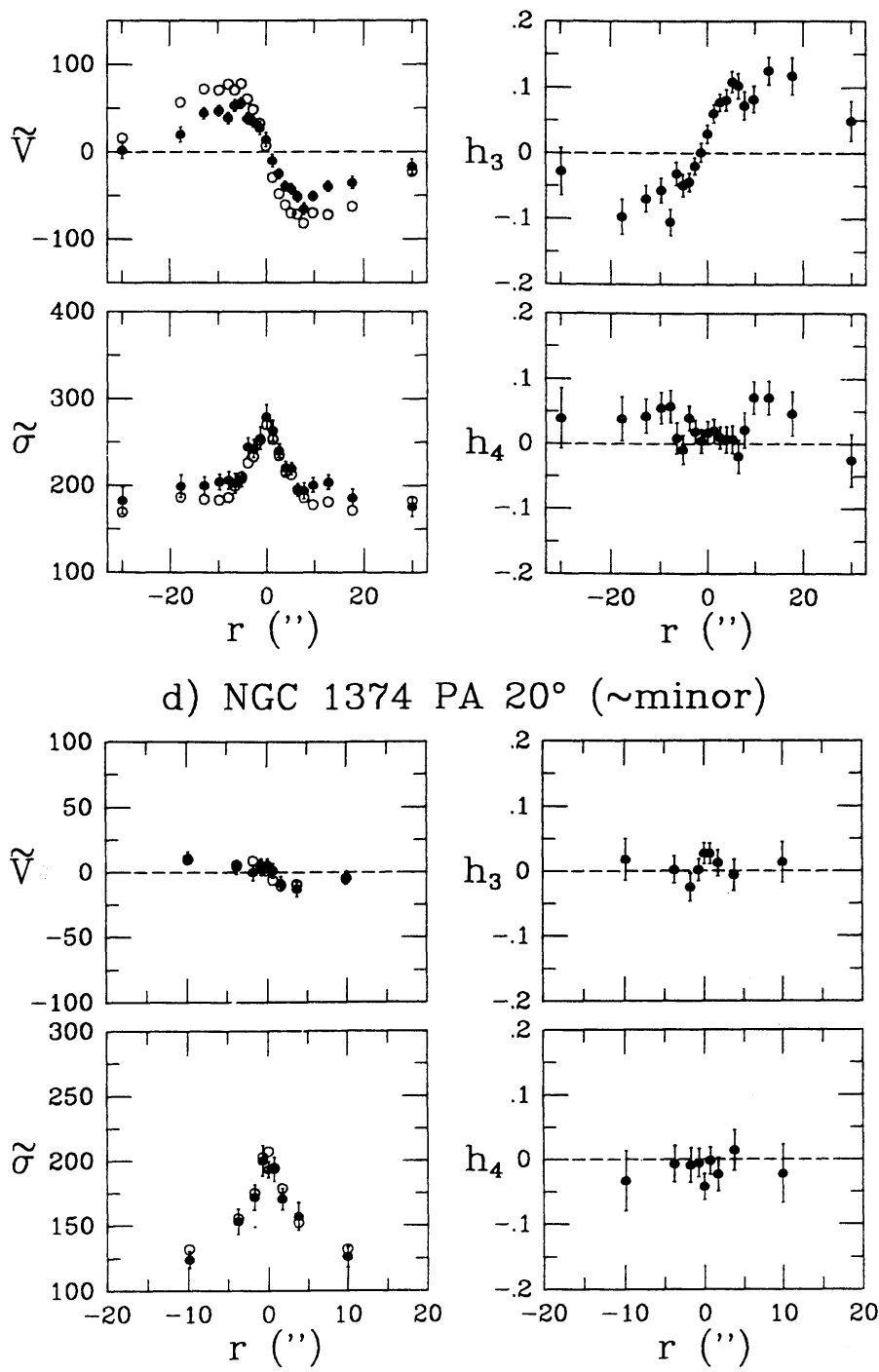

FIG. 4.-Kinematical quantities for our program galaxies. Line profiles of the form eq. (9) were fitted to the data, yielding parameters $\left(\gamma, V, \sigma, h_{3}, h_{4}\right)$. From these quantities improved estimates $(\tilde{\gamma}, \tilde{V}, \tilde{\sigma})$ for the true line strength, mean velocity, and velocity dispersion were derived as described in $\S 2.4$. The quantities $\tilde{V}, \tilde{\sigma}, h_{3}$, and $h_{4}$ are plotted as solid circles in the four panels of each figure. Plotted for comparison as open circles (without error bars) in the panels for $\tilde{V}$ and $\tilde{\sigma}$ are the estimates for the mean velocity and velocity dispersion as obtained by fitting a Gaussian line profile to the data. Positive radii lie to the east. Plotted for comparison as open squares in the right two panels of Fig. $4 a$ are the results of applying our new method to the old data for the major axis of IC 1459 presented previously by FI. Notice that the results agree well, even though the old data were taken with a different telescope, at lower spatial and instrumental resolution.

TABLE 3

Characteristics of the Data Acquisition and Reduction

\begin{tabular}{|c|c|c|c|}
\hline Characteristic & IC 1459 & NGC 1374 & NGC 4278 \\
\hline Date & 1988 Nov & 1988 Nov & $1991 \mathrm{Feb}$ \\
\hline Telescope. & CTIO $4 \mathrm{~m}$ & CTIO $4 \mathrm{~m}$ & KPNO $4 \mathrm{~m}$ \\
\hline Spectrograph & RC Spectrograph & RC Spectrograph & RC Spectrograph \\
\hline Exposure time (minutes) & 30 & 45,50 & 90 \\
\hline Seeing FWHM ... & 1.5 & 0.9 & $1^{\prime \prime}-1 " .5$ \\
\hline Dispersion $\left(\AA\right.$ pixel $\left.^{-1}\right)$ & 1.4 & 1.4 & 0.9 \\
\hline Scale $\left(\operatorname{arcsec}\right.$ pixel $\left.^{-1}\right)$ & 0.73 & 0.73 & 1.3 \\
\hline Spectral resolution $(\sigma)\left(\mathrm{km} \mathrm{s}^{-1}\right)$. & 60 & 80 & 100 \\
\hline Slit position angle .............. & $39^{\circ}$ & $110^{\circ}, 20^{\circ}$ & $19^{\circ}$ \\
\hline Major axis position angle ${ }^{\mathrm{a}}$ & $35^{\circ}$ & $120^{\circ}$ & $16^{\circ}$ \\
\hline Wavelength range used in Fourier fit $(\AA)$. & $4767-5496$ & $4767-5496$ & $4919-5374$ \\
\hline
\end{tabular}

a The major axis position angles for IC 1459 and NGC 4278 are those at a galactocentric distance of $10^{\prime \prime}$, as taken from the CCD surface photometry of Franx et al. 1989b and Peletier et al. 1990, respectively. The major axis position angle of NGC 1374 was taken from Lauberts \& Valentijn 1989. 


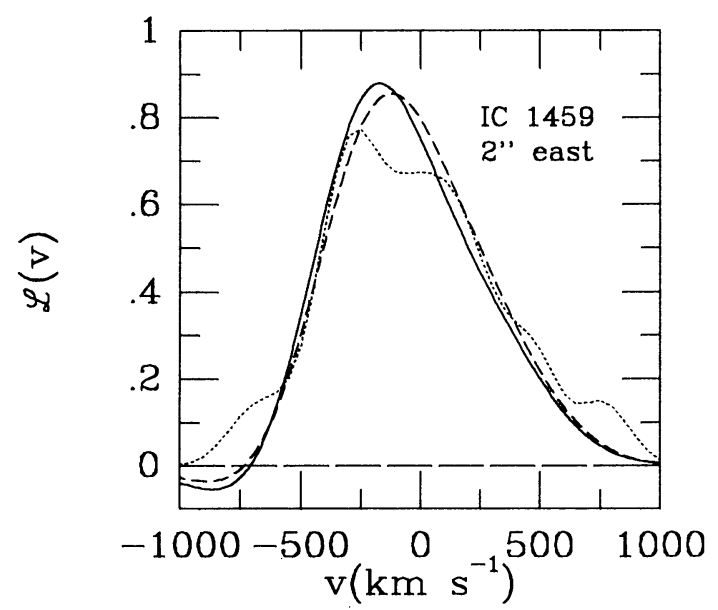

Fig. 5.-Major axis line profile of IC $14592^{\prime \prime}$ east of the center. The solid curve is the line profile derived from the high-resolution data presented here. The dashed curve is the line profile derived from the low-resolution data presented previously by FI. The dotted curve is the line profile derived from the low-resolution data by FI, using a modification of the CLEAN algorithm. The curves agree well. The normalization is arbitrary.

below). The large amplitude of $h_{3}(\sim 0.14)$ in Figure $4 a$ confirms these results. The higher order terms $h_{5}$ and $h_{6}$ were also determined. The resulting profiles (not plotted here) are noisy, but clearly have lower amplitude than $h_{3}$. This again justifies the use of only the first few terms in the series expansion equation (9).

We have also reanalyzed with our new method some major-axis data for IC 1459 presented previously by FI. These old data were taken with the ESO $2.2 \mathrm{~m}$ telescope at lower spectral resolution $\left(\sigma \approx 100 \mathrm{~km} \mathrm{~s}^{-1}\right)$ and lower spatial resolution $\left(1.8\right.$ pixel $\left.{ }^{-1}\right)$ than the data presented here (compare Table 3). The results for $h_{3}$ and $h_{4}$ for the old data are plotted as open squares in Figure $4 a$. It is comforting that the results for the two data sets agree so well. The $h_{3}$ profile has a somewhat smaller amplitude in the old data due to the poorer spatial resolution. Figure 5 plots the major axis line profile $2^{\prime \prime}$ east of the nucleus. The solid curve is the line profile derived from the new data, the dashed curve is the line profile derived from the old data. The latter line profile has more stars at higher velocities, as a consequence of the poorer spatial resolution. Plotted for comparison as a dotted curve is the line profile derived from the old data by FI, using a modification of the CLEAN algorithm known from radio astronomy. Comparison of the two line profiles derived from the old data suggests that the CLEAN method has erroneously assigned some of the power at low velocities to the wings of the line profile. The method presented here is thus to be preferred over the CLEAN method, also because the error analysis is much more straightforward.

NGC 4278 has a peculiar rotation curve discussed before by Davies \& Birkinshaw (1988) and Illingworth \& Franx (1989), and confirmed here (Fig. $4 b$ ). Beyond a galactocentric distance of $\sim 30^{\prime \prime}$ the rotation curve drops to zero, or even changes sign. This might be related to the large isophote twist in NGC 4278 (Peletier et al. 1990). The line profiles on the major axis are asymmetric. The $h_{3}$ profile has an amplitude of $\sim 0.11$, and reaches its maximum far from the center, at $\sim 15^{\prime \prime}$. The quantity $h_{4}$ rises by $\sim 0.05$ at $\sim 7$. This is at approximately the same galactocentric distance, where the rotation curve stops rising and the velocity dispersion stops falling.

Also NGC 1374 has asymmetric line profiles on its major axis (Fig. $4 c$ ). However, the amplitude of $h_{3}(\sim 0.08)$ is smaller than in IC 1459 and NGC 4278. On the minor axis (Fig. 4d) $h_{3} \approx 0$, as it should be. The profile of $h_{4}$ on the major axis has a maximum at $\sim 2$.

For all three galaxies the amplitude of $h_{3}$ is too large to be due to the effects of projection along the line-of-sight (stars well in front and behind the tangent point tend to add a low-velocity tail to the line profile), see, e.g., the calculations by Bender (1990). Consequently, either a kinematically distinct subcomponent must be present, the distribution of stars over azimuthal velocities $v_{\phi}$ must be significantly skew (as is the case in the simple rotating models discussed in $\S 3$ ), or both. Such kinematical signatures can arise in a variety of scenarios (e.g., FI; Balcells 1991), and contain information on the structure and history of these systems.

Plotted for comparison as open circles in Figures $4 a-4 d$ are the estimates of the rotation velocity and velocity dispersion obtained by fitting Gaussian line profiles to the data. Clearly these estimates contain a variety of systematic errors. The true amplitude of the major axis rotation curves are overestimated by $30 \%$ or more due to the asymmetry of the major axis line profiles (compare eq. [18]). The estimates of the velocity dispersion are influenced by trends in $h_{4}$ with radius. For example, upon fitting Gaussian line profiles to the major axis of NGC 1374 (Fig. $4 c$; open circles), one would conclude that the velocity dispersion at $\sim 2^{\prime \prime}$ is $\sim 50 \mathrm{~km} \mathrm{~s}$ lower than in the center. This, however, is incorrect, and is only a consequence of the maximum in $h_{4}$ at $\sim 2^{\prime \prime}$, which causes the velocity dispersion at $\sim 2^{\prime \prime}$ to be somewhat underestimated with respect to the central velocity dispersion (cf. eq. [18]). Indeed $\tilde{\sigma}(r)$ (solid circles), which is a better estimate of the true velocity dispersion, falls off much more smoothly away from the center.

Variations of $h_{4}$ with radius are uninfluenced by artificial constant offsets like those discussed in $\S 4$. They can arise through variations of the coefficient of kurtosis of the line profile with radius. However, other effects can also contribute. First, in the galaxies with a steep central rotation velocity gradient, seeing smearing will tend to make the central line profile more flat-topped. Second, in a galaxy with a kinematically distinct subcomponent (assumed to have higher rotation and lower dispersion than the primary component), the line profile will become more flat-topped as the relative velocity between the two components increases. Detailed modeling of these two effects, that both tend to decrease $h_{4}$, is required to fully understand the results for IC 1459 and NGC1374. In NGC 4278 seeing cannot cause the central depression in $h_{4}$, as the rotation curve is not particularly steep. It is thus more likely due to a real variation of the kurtosis of the line profile with galactocentric distance. 


\section{CONCLUSIONS}

To interpret long-slit spectra of elliptical galaxies it is usually assumed that the line-of-sight velocity distribution, or line profile, is Gaussian. In the present paper a new parameterizaton is proposed, the Gauss-Hermite series (eq. [9]), which expands the line profile as a sum of orthogonal functions. This approach exploits the fact that Gaussians provide good low-order fits to observed line profiles. The use of orthogonal functions minimizes correlations between the errors in the parameters of the fit. This constitutes a major advantage of our parameterization over most alternatives. Our method yields estimates $\tilde{\gamma}$ of the line strength, $\widetilde{V}$ of the mean radial velocity, and $\tilde{\sigma}$ of the velocity dispersion, as well as two extra parameters, $h_{3}$ and $h_{4}$, that describe asymmetric and symmetric deviations of the line profile from a Gaussian, respectively. The new parameterization can easily be built into existing programs for the interpretation of long-slit spectra.

Model calculations for the outer parts of spherical galaxies yield line profiles that deviate significantly from Gaussians. Even for models with only mild velocity dispersion anisotropy the rms deviations from a Gaussian can be of order $10 \%$. By fitting Gaussians to these line profiles one obtains estimates for the rotation velocity and velocity dispersion that can be in error by $10 \%$ or more. The quantities $\tilde{V}$ and $\tilde{\sigma}$ obtained with the new method are indeed much better estimates of the true mean radial velocity and velocity dispersion.

The new parameterization was built into the Fourier fitting program described by Franx et al. (1989a), and applied to long-slit spectroscopic observations of IC 1459, NGC 1374, and NGC 4278. For these intermediate spectral resolution, high S/N observations, $h_{3}$ can be satisfactorily determined. The profile of $h_{4}$ along the slit can only be determined up to a small constant offset due to the problems associated with continuum subtraction and the choice of template spectrum. Variations of $h_{4}$ with galactocentric distance are unaffected by these problems. Results for $h_{3}$ and $h_{4}$ on the major axis of IC 1459 obtained with different telescopes and spectral resolution agree well.

All three galaxies have asymmetric line profiles on their major axis, with the asymmetry changing sign upon going from one side of the nucleus to the other (such that the tail of the line profile is always away from the direction of rotation). In IC 1459 the asymmetry is related to the counter-rotating core component (Franx \& Illingworth 1988). Due to the asymmetry of the line profiles for these galaxies, the amplitude of the rotation curve is overestimated by $30 \%$ or more, if the line profiles are assumed Gaussian. In all three galaxies also symmetric deviations from a Gaussian are present that vary as a function of radius. In NGC 4278, for example, $h_{4}$ increases by $\sim 0.05$ at $7^{\prime \prime}$ from the center, approximately the same distance as where the rotation velocity stops rising and the velocity dispersion stops falling. Varying symmetric deviations from a Gaussian can be due to real variations of the coefficient of kurtosis of the line profile, to seeing smearing, or to the presence of a kinematically distinct subcomponent. Estimates of the velocity dispersion based on Gaussian line profiles can contain systematic errors that vary with radius. In NGC 1374 the assumption of Gaussian line profiles incorrectly yields that the velocity dispersion increases by $\sim 50 \mathrm{~km} \mathrm{~s}{ }^{-1}$ in the inner $2^{\prime \prime}$. Our new method demonstrates that this is not correct: $h_{4}$ has a maximum at $\sim 2$, and the velocity dispersion falls smoothly away from the center.

Elliptical galaxies clearly show a great variety in their line profile shapes. Modeling of observed line profiles might ultimately lead to a better understanding of their shape, structure, formation history, velocity dispersion anisotropy, and mass-to-light ratio.

The authors thank Tim de Zeeuw for discussions throughout this project, and the referee, Herwig Dejonghe, for useful comments. Garth Illingworth collaborated in collecting the data presented in $\S 4$. R.vdM. acknowledges support from the LKBF and NWO, and the hospitality of the CfA. M. F. was supported by Hubble Fellowship grant HF-1016.01-91A from the Space Telescope Science Institute, which is operated by AURA, Inc., under NASA contract NAS5-26555.

R.vdM. wishes to dedicate this paper to his mother, who died 1992 June 2 . Her smile lives on in everyone who knew her.

\section{APPENDIX A}

\section{HERMITE POLYNOMIALS}

This appendix discusses some properties of Hermite polynomials. A more detailed treatment on these polynomials and orthogonal polynomials in general can be found in, e.g., Abramowitz \& Stegun (1965). There exist two types of Hermite polynomials. In the present paper these are discriminated by the use of different symbols. Note that our normalization of the Hermite polynomials differs from that of Abramowitz and Stegun. ${ }^{3}$

Let the function $\alpha(y)$ be the standard Gaussian

$$
\alpha(y)=\frac{1}{\sqrt{2 \pi}} e^{-(1 / 2) y^{2}} .
$$

We define the first type of Hermite polynomials, $\mathscr{H}_{\mathrm{e}_{l}}(y)$, by the relation

$$
\left(-\frac{d}{d y}\right)^{l} \alpha(y)=\sqrt{l !} \mathscr{H}_{\mathrm{e}_{l}}(y) \alpha(y) .
$$

\footnotetext{
${ }^{3}$ The Hermite polynomials $\mathscr{H}_{\mathrm{e}_{l}}(y)$ and $H_{l}(y)$ defined here are related to the Hermite polynomials $\mathscr{H}_{\mathrm{e}_{\mathrm{l}}}[\mathrm{Abr}$. $](y)$ and $H_{l}[\mathrm{Abr}$.] $(y)$ defined by Abramowitz and Stegun through $\mathscr{H}_{\mathrm{e}_{1}}(y)=\mathscr{H}_{\mathrm{e}_{1}}[\mathrm{Abr}].(y) / \sqrt{l !}$ and $H_{l}(y)=H_{l}[\mathrm{Abr}].(y) / \sqrt{l ! 2^{l}}$.
} 
The function $\mathscr{H}_{\mathrm{e}_{l}}(y)$ is a polynomial of degree $l$ in $y$. The $\mathscr{H}_{\mathrm{e}_{l}}(y)$ are orthonormal with respect to the weight function $\alpha(y)$, i.e.,

$$
\int_{-\infty}^{\infty} \mathscr{H}_{\mathrm{e}_{m}}(y) \mathscr{H}_{\mathrm{e}_{n}}(y) \alpha(y) d y=\delta_{m n} .
$$

We define the second type of Hermite polynomials, $H_{l}(y)$, by the relation

$$
H_{l}(y)=\mathscr{H}_{\mathrm{e}}(y \sqrt{2}) .
$$

The first five Hermite polynomials of this type are

$$
\begin{gathered}
H_{0}(y)=1, \quad H_{1}(y)=\sqrt{2} y, \quad H_{2}(y)=\frac{1}{\sqrt{2}}\left(2 y^{2}-1\right), \quad H_{3}(y)=\frac{1}{\sqrt{6}}\left(2 \sqrt{2} y^{3}-3 \sqrt{2} y\right), \\
H_{4}(y)=\frac{1}{\sqrt{24}}\left(4 y^{4}-12 y^{2}+3\right) .
\end{gathered}
$$

From the identity $\alpha^{2}(y)=\alpha[y \sqrt{2}] / \sqrt{2 \pi}$ and equation (A3) it follows that

$$
\int_{-\infty}^{\infty} H_{m}(y) H_{n}(y) \alpha^{2}(y) d y=\frac{1}{2 \sqrt{\pi}} \delta_{m n} .
$$

The $H_{l}(y)$ are thus orthogonal with respect to the weight function $\alpha^{2}(y)$. The Fourier transform of $H_{l}(y) \alpha(y)$ is $(\mathrm{Gradshteyn} \& \operatorname{Ryzhik}$ 1980 , eq. [7.376.1])

$$
\int_{-\infty}^{\infty} H_{l}(y) \alpha(y) e^{2 \pi i f y} d y=\sqrt{2 \pi} i^{l} H_{l}(2 \pi f) \alpha(2 \pi f) .
$$

\section{APPENDIX B}

\section{LINE PROFILES FOR THE OUTER PARTS OF ELLIPTICAL GALAXIES}

\section{B1. PROJECTED VELOCITY MOMENTS FOR NONROTATING MODELS}

Projected velocity moments are calculated for the nonrotating models for the outer parts of elliptical galaxies described in $\S 3$. Line profiles such as those in Figures $2 a$ and $2 b$ can be reconstructed from these moments using the Gram-Charlier series of type A, discussed in $\S \mathrm{B} 2$. The line profiles for the case $\beta \rightarrow-\infty$ (circular orbits only) and $\beta \rightarrow 1$ (radial orbits only) plotted in Figures $2 a$ and $2 b$ were calculated analytically in a manner similar to Appendix C of Merritt (1987).

Let $(r, \theta, \phi)$ and $(R, \phi, z)$ be the usual spherical and cylindrical coordinates, respectively, with the $z$-axis toward the observer. The projected line-of-sight velocity moments are $\llbracket \overline{v_{z}^{n}} \rrbracket$. The overbar denotes the local average over velocities. The open brackets denote the mass-weighted projection along the line of sight. Since $f(v)=f(-v), \llbracket\left[v_{z}^{n} \rrbracket\right]=0$ for odd $n$. Henceforth $n$ is assumed to be an even integer. The moment $\llbracket \overline{v_{z}^{n}} \rrbracket$ is the quotient of the line-of-sight projection of $\rho v_{z}^{n}$ and the line-of-sight projection of $\rho$. By assumption $\rho(r)=C r^{-t}$ eq. [20]), and thus

$$
\llbracket \overline{v_{z}^{n}} \rrbracket=\frac{\int_{0}^{\pi / 2} \sin ^{t-2} \theta \overline{v_{z}^{n}}(r=\tau / \sin \theta) d \theta}{(1 / 2) \mathscr{B}\left[(t-1) / 2, \frac{1}{2}\right]},
$$

where $\tau$ is the projected galactocentric distance of the observations, and $\mathscr{B}(\cdot, \cdot)$ is the Beta function. Since $v_{z}=v_{r} \cos \theta-v_{\theta} \sin \theta$, and since $\overline{v_{r}^{i} v_{\theta}^{n-i}}=0$ for odd $i$, the binomial theorem yields that

$$
\overline{v_{z}^{n}}=\sum_{k=0}^{n / 2}\left(\begin{array}{c}
n \\
2 k
\end{array}\right) \cos ^{2 k} \theta \sin ^{n-2 k} \theta \overline{v_{r}^{2 k} v_{\theta}^{n-2 k}},
$$

where

$$
\overline{v_{r}^{2 i} v_{\theta}^{2 j}} \equiv \int v_{r}^{2 i} v_{\theta}^{2 j} f(\mathscr{E}, L) d^{3} \boldsymbol{v} / \int f(\mathscr{E}, L) d^{3} \boldsymbol{v}
$$

Upon substitution of $f(\mathscr{E}, L)$ from equation (19) the integrals in this definition can be evaluated by defining spherical coordinates in velocity space. The result is

$$
\overline{v_{r}^{2 i} v_{\theta}^{2 j}}=2^{i+j} \frac{(2 j-1) ! !}{(2 j) ! !} \frac{\mathscr{B}[i+(1 / 2), j-\beta+1]}{\mathscr{B}[(1 / 2),-\beta+1]} \frac{\int_{\mathscr{E}_{\text {min }}}^{\Psi} f_{0}(\mathscr{E})(\Psi-\mathscr{E})^{i+j+(1 / 2)-\beta} d \mathscr{E}}{\int_{\mathscr{E}_{\text {min }}}^{\Psi} f_{0}(\mathscr{E})(\Psi-\mathscr{E})^{(1 / 2)-\beta} d \mathscr{E}},
$$

where $\mathscr{E}_{\min }$ is the minimum binding energy for which a star is still bound to the system $\left(\mathscr{E}_{\min }=0\right.$ for a system of finite mass, and $\mathscr{E}_{\min }=-\infty$ for a system of infinite mass). ${ }^{4}$

\footnotetext{
${ }^{4}$ The expression for $\overline{v_{z}^{n}}$ implied by eqs. (B2) and (B4) could alternatively have been arrived at starting from eq. (A11) of Dejonghe (1987).
} 
In an elliptical galaxy that has no dark halo, the potential in the outer parts is approximately $\mathrm{Keplerian}$, i.e., $\Psi=G M / r$, where $M$ is the total mass of the system. For this potential $\mathscr{E}_{\min }=0$ and, with the use of equation (20),

$$
\frac{\int_{0}^{\Psi} f_{0}(\mathscr{E})(\Psi-\mathscr{E})^{i+j+(1 / 2)-\beta} d \mathscr{E}}{\int_{0}^{\Psi} f_{0}(\mathscr{E})(\Psi-\mathscr{E})^{(1 / 2)-\beta} d \mathscr{E}}=\frac{\Gamma[i+j+(3 / 2)-\beta]}{\Gamma[(3 / 2)-\beta]} \frac{\Gamma(t-2 \beta+1)}{\Gamma(t+i+j-2 \beta+1)}(G M / r)^{i+j}
$$

Evaluation of the integral in equation (B1) then yields after substitution of equations (B2), (B4), and (B5)

$$
\begin{array}{r}
\llbracket \overline{v_{z}^{n}} \rrbracket=\sum_{k=0}^{n / 2}\left(\begin{array}{c}
n \\
2 k
\end{array}\right) 2^{n / 2} \frac{\mathscr{B}\{(1 / 2)[t-1+(n / 2)+n-2 k],(1 / 2)+k\}}{\mathscr{B}[(1 / 2)(t-1),(1 / 2)]} \frac{(n-2 k-1) ! !}{(n-2 k) ! !} \frac{\mathscr{B}[k+(1 / 2),(n / 2)-k-\beta+1]}{\mathscr{B}[(1 / 2),-\beta+1]} \\
\times \frac{\Gamma[(n / 2)+(3 / 2)-\beta]}{\Gamma[(3 / 2)-\beta]} \frac{\Gamma(t-2 \beta+1)}{\Gamma[t+(n / 2)-2 \beta+1]},
\end{array}
$$

where $\sqrt{G M / \tau}$, the circular velocity at radius $\tau$, has been introduced as unit of velocity. For $n=2$ this expression reduces to

$$
\llbracket \overline{v_{z}^{2}} \rrbracket=\frac{t-1}{2(t+1)}\left\{\frac{\Gamma(t / 2)}{\Gamma[(t+1) / 2]}\right\}^{2}\left[\frac{t(1-\beta)+1}{2(1-\beta)+(t-1)}\right],
$$

and for $n=4$ to

$$
\llbracket \overline{v_{z}^{4}} \rrbracket=\frac{(t-1)}{t(t+2)(t+4)} \frac{(1-\beta)^{2}[(3 / 2)(t+1)(t+3)]+(1-\beta)[(3 / 2)(t+1)(t+7)]+9}{4(1-\beta)^{2}+(4 t-2)(1-\beta)+t(t-1)} .
$$

For $t=5$ these equations reproduce the results for the anisotropic Plummer models of Dejonghe (1987) in the limit of large radii.

In an elliptical galaxy in which the potential in the outer parts is dominated by a dark halo, it is more appropriate to adopt for $\Psi$ the logarithmic potential $\Psi=-V_{c}^{2} \ln r$. For this potential $\mathscr{E}_{\min }=-\infty$ and, with the use of equation (20),

$$
\frac{\int_{-\infty}^{\Psi} f_{0}(\mathscr{E})(\Psi-\mathscr{E})^{i+j+(1 / 2)-\beta} d \mathscr{E}}{\int_{-\infty}^{\Psi} f_{0}(\mathscr{E})(\Psi-\mathscr{E})^{(1 / 2)-\beta} d \mathscr{E}}=\frac{\Gamma[i+j+(3 / 2)-\beta]}{\Gamma[(3 / 2)-\beta]}\left(\frac{V_{c}^{2}}{t-2 \beta}\right)^{i+j}
$$

Evaluation of the integral in equation (B1) then yields after substitution of equations (B2), (B4), and (B7)

$$
\begin{gathered}
\llbracket \overline{v_{z}^{n}} \rrbracket=\sum_{k=0}^{n / 2}\left(\begin{array}{c}
n \\
2 k
\end{array}\right) \frac{\mathscr{B}[(1 / 2)(t-1+n-2 k),(1 / 2)+k]}{\mathscr{B}[(1 / 2)(t-1),(1 / 2)]} \frac{(n-2 k-1) ! !}{(n-2 k) ! !} \frac{\mathscr{B}[k+(1 / 2),(n / 2)-k-\beta+1]}{\mathscr{B}[(1 / 2),-\beta+1]} \\
\times \frac{\Gamma[(n / 2)+(3 / 2)-\beta]}{\Gamma[(3 / 2)-\beta]}\left(\frac{2}{t-2 \beta}\right)^{n / 2},
\end{gathered}
$$

where $V_{c}$ has been introduced as unit of velocity. For $n=2$ this expression reduces to

$$
\llbracket \overline{v_{z}^{2}} \rrbracket=\frac{1}{t}\left[\frac{(1-\beta)(t-1)+1}{2(1-\beta)+(t-2)}\right]
$$

and for $n=4$ to

$$
\llbracket \overline{v_{z}^{4}} \rrbracket=\frac{1}{4 t(t+2)} \frac{(1-\beta)^{2}[(3 / 2)(t-1)(t+1)]+(1-\beta)[(3 / 2)(t-1)(t+5)]+9}{(1-\beta)^{2}+(1-\beta)(t-2)+(1 / 4)(t-2)^{2}} .
$$

In Figure 6 the velocity dispersion $\sigma_{0} \equiv \llbracket \overline{v_{z}^{2}} \rrbracket^{1 / 2}$, and coefficient of kurtosis $\xi_{2} \equiv \llbracket \overline{v_{z}^{4}} \rrbracket / \llbracket \overline{v_{z}^{2}} \rrbracket^{2}$ are plotted as function of $\beta$ for the case $t=4$, for both the Kepler and the logarithmic potential. Since radial motion in the outer parts is predominantly perpendicular to the line of sight the velocity dispersion decreases with $\beta$, whereas the coefficient of kurtosis tends to increase with $\beta$.

\section{B2. THE GRAM-CHARLIER SERIES OF TYPE A}

The so-called Gram-Charlier series of type $A$ is a useful tool in reconstructing a function for which all the moments are known. Criteria sufficient for convergence are given by Kendall \& Stuart (1943). For a normalized line profile $\mathscr{L}_{0}(v)$ with mean velocity $V_{0}$ and velocity dispersion $\sigma_{0}$ the series is

$$
\begin{aligned}
\mathscr{L}_{0}(v) & =\left[\alpha\left(w_{0}\right) / \sigma_{0}\right] \sum_{j=0}^{\infty} d_{j} \mathscr{H}_{\mathrm{e}_{j}}\left(w_{0}\right), \quad w_{0} \equiv\left(v-V_{0}\right) / \sigma_{0}, \\
d_{l} & =\int_{-\infty}^{\infty} \mathscr{L}_{0}(v) \mathscr{H}_{\mathrm{e}_{l}}\left(w_{0}\right) d v,
\end{aligned}
$$

where $\alpha(y)$ is defined in equation (4) and the $\mathscr{H}_{\mathrm{e}_{l}}(y)$ are Hermite polynomials as defined in Appendix A. The first five coefficients are

$$
d_{0}=1, \quad d_{1}=0, \quad d_{2}=0, \quad d_{3}=\xi_{1} / \sqrt{6}, \quad d_{4}=\left(\xi_{2}-3\right) / \sqrt{24},
$$

where $\xi_{1}$ and $\xi_{2}$ are the coefficients of skewness and kurtosis defined in $\S 2.4$. Note both the resemblance and the difference between the Gram-Charlier series of type A and the parameterization employed in the present paper $(\S 2.2)$. 

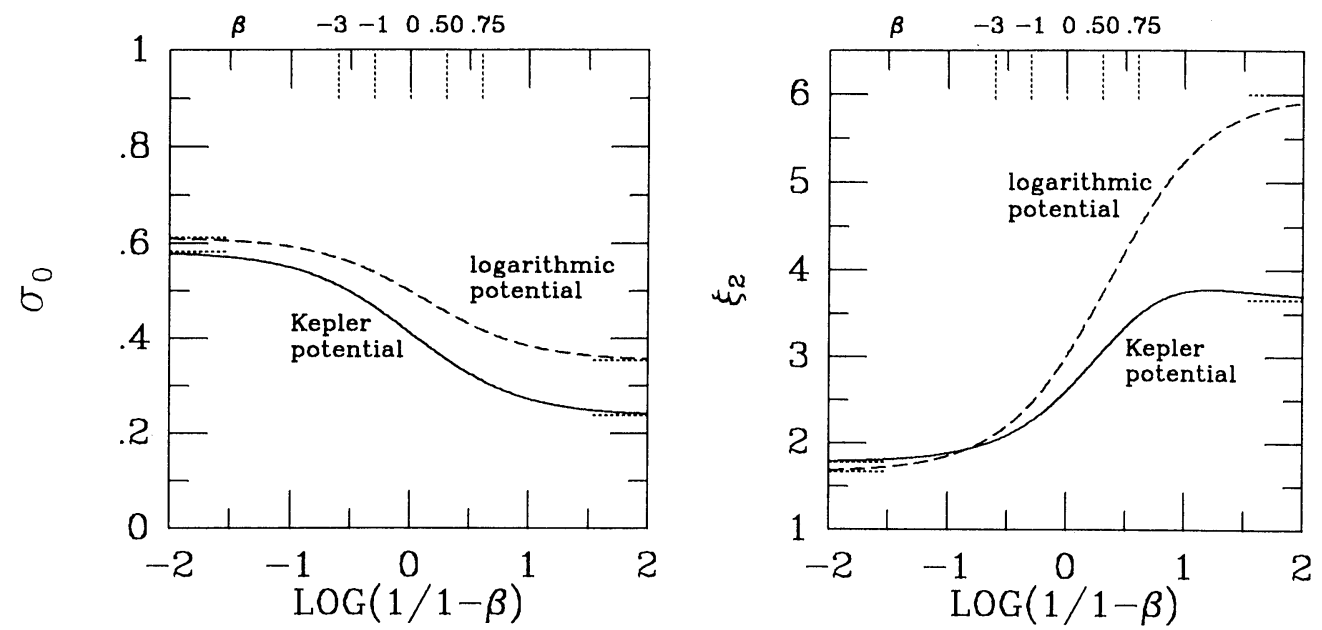

FIG. 6.-Plotted are the velocity dispersion $\sigma_{0}$ (left panel) and coefficient of kurtosis $\xi_{2}$ (right panel) as a function of $\log [1 /(1-\beta)]$ for nonrotating models for the outer parts of spherical galaxies in which the stellar mass density is $\rho \propto r^{-4}$, the potential is either Keplerian (solid curves) or logarithmic (dashed curves), and $\beta$ is the (constant) velocity dispersion anisotropy. The velocity dispersion $\sigma_{0}$ is given in units of the circular velocity at the galactocentric distance of the observations. The short horizontal dotted lines indicate asymptotic values for $\beta \rightarrow-\infty$ and $\beta \rightarrow 1$.

\section{B3. LINE PROFILES FOR SIMPLE ROTATING MODELS}

Consider a nonrotating model for the outer parts of elliptical galaxies in which the relative potential is logarithmic, $\Psi=$ $-V_{c}^{2} \ln r$, the stellar mass density is $\rho \propto r^{-4}$, and the stellar velocity distribution is isotropic. The distribution function of the stars is then

$$
f(\mathscr{E}) \propto\left[1 / 2 \pi\left(V_{c} / 2\right)^{2}\right]^{3 / 2} e^{\mathscr{E} /\left(V_{c} / 2\right)^{2}},
$$

where $\mathscr{E} \equiv \Psi(r)-\frac{1}{2} v^{2}$. This is a special case of the models discussed in $\S 3$ and $\S$ B1. By reversing stellar velocities such that in every point a fraction $\zeta$ of the stars has positive $v_{\phi}$ and a fraction $1-\zeta$ has negative $v_{\phi}$, the mass density remains unaltered, but the distribution function becomes

$$
f\left(\mathscr{E}, v_{\phi}\right) \propto\left\{\begin{array}{ll}
2 \zeta\left[1 / 2 \pi\left(V_{c} / 2\right)^{2}\right]^{3 / 2} \mathrm{e}^{\mathscr{E} /\left(V_{c} / 2\right)^{2}} & \left(v_{\phi} \geq 0\right) \\
2(1-\zeta)\left[1 / 2 \pi\left(V_{c} / 2\right)^{2}\right]^{3 / 2} e^{\mathscr{E} /\left(V_{c} / 2\right)^{2}} & \left(v_{\phi}<0\right)
\end{array} .\right.
$$

The observed line profile $\mathscr{L}_{0}(v)$ is obtained by first integrating over the velocity components perpendicular to the line of sight, and then along the line of sight. Assuming that the system is viewed from within the equatorial plane this yields

$$
\mathscr{L}_{0}(v)=\frac{1}{\sqrt{2 \pi}} e^{-(1 / 2) v^{2}}\left\{1+(2 \zeta-1)\left(\frac{4}{\pi \sqrt{2 \pi}}\right) \int_{0}^{\infty} e^{-(1 / 2) y^{2}}\left[\arctan \left(\frac{v}{y}\right)+\frac{(v / y)}{1+(v / y)^{2}}\right] d y\right\},
$$

where $V_{c} / 2$ has been introduced as unit of velocity. In Figure $2 c$ this line profile is plotted for several values of $\zeta$. Similar results can be derived for the case in which the mass density of the stars is, e.g., $\rho \propto r^{-3}$.

After submission of this article we found out that Gerhard (1993) had independently used the Gauss-Hermite series to describe the line profiles of theoretical models for elliptical galaxies. He discusses quantities $s_{l}$ that are obtained from equation (7) upon subsitution of $s_{l}$ for $h_{l}, 1$ for $\gamma_{0} / \gamma$, and the true dispersion of the line profile for $\sigma$. The difference in our approaches thus lies in the "scale" $\sigma$ of the Gauss-Hermite expansion equation (5): we use the dispersion of the best Gaussian fit to the line profile, whereas Gerhard uses the true dispersion of the line profile. The former quantity is better defined observationally, the latter quantity is easier to calculate for theoretical models. For data analysis the method presented here has the advantage that the error analysis is straightforward. There is no simple relation between our $h_{l}$ and Gerhard's $s_{l}$, but for any given theoretical line profile both are easily calculated. For real data one way to estimate the $s_{l}$ is to first determine $(\tilde{\gamma}, \tilde{V}, \tilde{\sigma})$ as described in the present paper, and then fit a function of the form of equation (5) to the data, with $(\gamma, V, \sigma)$ held fixed to $(\tilde{\gamma}, \tilde{V}, \tilde{\sigma})$.

\section{REFERENCES}

Abramowitz, M., \& Stegun, I. A. 1965, Handbook of Mathematical Functions (NY: Dover)

Balcells, M. 1991, A\&A, 249, L9

Bender, R. 1990, A\&A, 229, 441

Binney, J. J., \& Tremaine, S. 1987, Galactic Dynamics (Princeton: Princeton Univ. Press)

Cayrel de Strobel, G., Bentolila, C., Hauck, B., \& Duquennoy, A. 1985, A\&AS, 59,145

Davies, R. L., \& Birkenshaw, M. 1988, ApJS, 68, 409

Dejonghe, H. 1987, MNRAS, 224, 13
Dejonghe, H., \& Merrit, D. R. 1992, ApJ, 391, 531

de Zeeuw, P. T., \& Franx, M. 1991, ARA\&A, 29, 239

Evans, N. W. 1993, MNRAS, 260,191

Franx, M., \& Illingworth, G. D. 1988, ApJ, 327, L55 (FI)

Franx, M., Illingworth, G. D., \& Heckman, T. M. 1989a, ApJ, 344, 613 (FIHa) $1989 \mathrm{~b}, \mathrm{AJ}, 98,538$

Gerhard, O. E. 1991, MNRAS, 250, 812

. 1993, MNRAS, submitted

Gradshteyn, I. S., \& Ryzhik, I. M. 1980, Tables of Integrals, Series, and Products (San Diego: Academic Press) 
1.

it Hénon, M. 1973, A\&A, 24, 229

'' Illingworth, G. D., \& Franx, M. 1989, in Dynamics of Dense Stellar Systems,

ed. D. Merritt (Cambridge: Cambridge Univ. Press), 13

I $m$ ' Kendall, M. G., \& Staurt, A. 1943, The Advanced Theory of Statistics, Vol. 1

I.I (London: Charles Griffin \& Co.)

I

Larsen, N., Nørgaard-Nielsen, H. U., Kjærgaard, P., \& Dickens, R. J. 1983, A\&A, 117,257

Lauberts, A., \& Valentijn, E. A. 1989, The Surface Photometry Catalogue of the ESO-Uppsala Galaxies (Garching: ESO)

Merrifield, M. R., \& Kent, S. M. 1990, AJ, 99, 1548

Merrit, D. R. 1987, ApJ, 313, 121

Myller-Lebedeff, W. 1908, Math. Ann. 64, 388

Peletier, R. F., Davies, R. L., Illingworth, G. D., Davies, L. E., \& Cawson, M.

Pickles, A. J. 1985a, ApJS, 59, 33

$1985 \mathrm{~b}$, ApJ, 296, 340

Press, W. H., Flannery, P., Teukolsky, S. A., \& Vetterling, W. T. 1986, Numerical Recipes (Cambridge: Cambridge Univ. Press)

Rix, H., \& White, S. D. M. 1990, ApJ, 362, 52 1992, MNRAS, 254, 389 (RW)

Sargent, W. L. W., Schechter, P. L., Boksenberg, A., \& Shortridge, K. 1977 ApJ, 212, 326

Simkin, S. M. 1974, A\&A, 31, 129

Tonry, J. L., \& Davis, M. 1979, AJ, 84, 1511

Toomre, A. 1982, ApJ, 259, 535

Winsall, M. L., \& Freeman, K. C. 1993a, A\&A, submitted 1990, AJ, 100, 1091 